\title{
Evolution of the $F_{0} F_{1}$ ATP Synthase Complex in Light of the Patchy Distribution of Different Bioenergetic Pathways across Prokaryotes
}

\author{
Vassiliki Lila Koumandou, Sophia Kossida*
}

Bioinformatics \& Medical Informatics Team, Biomedical Research Foundation, Academy of Athens, Athens, Greece

\begin{abstract}
Bacteria and archaea are characterized by an amazing metabolic diversity, which allows them to persist in diverse and often extreme habitats. Apart from oxygenic photosynthesis and oxidative phosphorylation, well-studied processes from chloroplasts and mitochondria of plants and animals, prokaryotes utilize various chemo- or lithotrophic modes, such as anoxygenic photosynthesis, iron oxidation and reduction, sulfate reduction, and methanogenesis. Most bioenergetic pathways have a similar general structure, with an electron transport chain composed of protein complexes acting as electron donors and acceptors, as well as a central cytochrome complex, mobile electron carriers, and an ATP synthase. While each pathway has been studied in considerable detail in isolation, not much is known about their relative evolutionary relationships. Wanting to address how this metabolic diversity evolved, we mapped the distribution of nine bioenergetic modes on a phylogenetic tree based on 16S rRNA sequences from 272 species representing the full diversity of prokaryotic lineages. This highlights the patchy distribution of many pathways across different lineages, and suggests either up to 26 independent origins or 17 horizontal gene transfer events. Next, we used comparative genomics and phylogenetic analysis of all subunits of the $F_{0} F_{1}$ ATP synthase, common to most bacterial lineages regardless of their bioenergetic mode. Our results indicate an ancient origin of this protein complex, and no clustering based on bioenergetic mode, which suggests that no special modifications are needed for the ATP synthase to work with different electron transport chains. Moreover, examination of the ATP synthase genetic locus indicates various gene rearrangements in the different bacterial lineages, ancient duplications of atpl and of the beta subunit of the $\mathrm{F}_{0}$ subcomplex, as well as more recent stochastic lineage-specific and species-specific duplications of all subunits. We discuss the implications of the overall pattern of conservation and flexibility of the $F_{0} F_{1}$ ATP synthase genetic locus.
\end{abstract}

Citation: Koumandou VL, Kossida S (2014) Evolution of the $F_{0} F_{1}$ ATP Synthase Complex in Light of the Patchy Distribution of Different Bioenergetic Pathways across Prokaryotes. PLoS Comput Biol 10(9): e1003821. doi:10.1371/journal.pcbi.1003821

Editor: Christos A. Ouzounis, Hellas, Greece

Received January 29, 2014; Accepted July 18, 2014; Published September 4, 2014

Copyright: () 2014 Koumandou, Kossida. This is an open-access article distributed under the terms of the Creative Commons Attribution License, which permits unrestricted use, distribution, and reproduction in any medium, provided the original author and source are credited.

Funding: This research was supported by a Marie Curie Intra European Fellowship within the 7th European Community Framework Programme (FP7-PEOPLE2011-IEF proposal $N^{\circ}$ 298890). The funders had no role in study design, data collection and analysis, decision to publish, or preparation of the manuscript.

Competing Interests: The authors have declared that no competing interests exist.

*Email: skossida@bioacademy.gr

\section{Introduction}

Bacteria and archaea use diverse bioenergetic electron transport chains to generate ATP. Apart from photosynthesis and aerobic respiration, many other bacterial and archaeal bioenergetic pathways have been characterized in considerable biochemical detail (e.g. $[1,2,3,4,5,6,7,8,9,10,11,12])$. However, the origins of the diversity of bioenergetic pathways, and their evolutionary relationships, have so far received relatively little attention. Did each pathway evolve independently or did they all evolve from a common ancestral metabolic mode? As in organismal evolution, it is likely that there were some novel innovations and that parts of pre-existing pathways were co-opted to evolve into new pathways. Molecular evolutionary studies of shared proteins amongst prokaryotes, coupled to data from the geological record, indicate that the vast majority of extant bioenergetic pathways evolved within the first billion years from the origin of life on earth $[13,14]$ and have since been mostly characterized by stasis [15]. Interestingly, when $16 \mathrm{~S}$ rRNA phylogenetic analysis is carried out for a variety of prokaryotes, organisms that utilize different bioenergetic pathways don't group into clear monophyletic groups, i.e. closely related organisms can utilize quite distinct bioenergetic strategies [16,17]. This may be due to horizontal gene transfer [18], and highlights the challenge of deciphering the evolution of these pathways.

While most previous studies have focused on comparison of the organisms that harbour the bioenergetic machinery, direct comparisons of the proteins that compose the bioenergetic machinery has been more limited. Most bioenergetic pathways use an electron transport chain (ETC) to generate a proton gradient across the membrane, and the energy released by the flow of electrons to compensate for this gradient is then used by the ATP synthase to generate ATP. The electron transport chains of disparate pathways have a similar general structure, being composed of protein complexes acting as electron donors and acceptors, with a central cytochrome $b c$-type complex and mobile electron carriers between them. Three scenarios are envisaged for the early evolution of energetic flexibility in the bacteria and the archaea: (i) each bioenergetic pathway evolved independently, (ii) all bioenergetic pathways evolved from a "simpler" ancestral metabolism, (iii) some new metabolic capabilities evolved by the modification of pre-existing pathways. The third scenario is the 


\section{Author Summary}

Bacteria and archaea are the most primitive forms of life on Earth, invisible to the naked eye and not extremely varied or impressive in their appearance. Nevertheless, they are characterized by an amazing metabolic diversity, especially in the different processes they use to generate energy in the form of ATP. This allows them to persist in diverse and often extreme habitats. Wanting to address how this metabolic diversity evolved, we mapped the distribution of nine bioenergetic modes across all the major lineages of bacteria and archaea. We find a patchy distribution of the different pathways, which suggests either frequent innovations, or gene transfer between unrelated species. We also examined the F-type ATP synthase, a protein complex which is central to all bioenergetic processes, and common to most types of bacteria regardless of how they harness energy from their environment. Our results indicate an ancient origin for this protein complex, and suggest that different species, without necessitating major innovation, used their pre-existing ATP synthase and adapted it to work with different bioenergetic pathways. We also describe gene duplications and rearrangements of the ATP synthase subunits in different lineages, which suggest further flexibility and robustness in the control of ATP synthesis.

most likely, and has been highlighted through detailed analysis of the bioenergetic protein complexes, e.g. for oxygenic and anoxygenic photosynthesis [19,20,21].

The unprecedented availability of genomic data enables us to address evolutionary questions relating to the events that led to the emergence of this metabolic diversity early in the evolution of life on Earth. Although various studies have looked at the evolution of ATP synthases across the bacteria and the archaea (e.g. $[22,23,24])$, these have mostly addressed the relative relationships between the F-V- and A-type ATPases, and no study has looked at organisms spanning the full bioenergetic diversity of bacteria. We chose to examine the $\mathrm{F}_{0} \mathrm{~F}_{1}$ ATP synthase complex, common to nine bioenergetic modes, and sampled a large variety of species across all major lineages to establish their homology and evolutionary relationships. We first asked whether the evolution of the ATP synthase complexes in these species agrees with the $16 \mathrm{~S}$ rRNA phylogeny, i.e. whether they cluster according to the type of ETC, or based on taxonomic groups. This enables us to check for horizontal gene transfer events concerning the ATP synthase, as well as for putative specific modifications in the ATP synthase subunits associated with each bioenergetic mode. We also examined the structure of the $\mathrm{F}_{0} \mathrm{~F}_{1}$ ATP synthase genetic locus, and report a variety of both ancient and recent gene duplications and rearrangements.

\section{Results}

\section{No monophyly of bioenergetic modes}

In this study, we focused on nine pathways most of which have been well characterized at the biochemical level, and for which enough sequence information is available to enable assessment of the diversity within each group as well as inter-group relationships:

(i) Oxygenic photosynthesis (cyanobacteria, e.g. Synechococcus)

(ii) Anoxygenic photosynthesis (green sulfur bacteria, e.g. Chlorobium; green non-sulfur bacteria, e.g. Chloroflexus; proteobacteria, e.g. Chromatium, Rhodospirillum, Rhodopseudomonas; heliobacteria, e.g. Heliobacterium)

(iii) Methanogenesis (methanogenic archaea, e.g. Methanosarcina, Methanococcus)

(iv) Sulfate reduction (bacteria, e.g. Desulfovibrio, and archaea, e.g. Archaeoglobus)

(v) Sulfur reduction (bacteria, e.g. Sulfurospirillum, and archaea, e.g. Ignicoccus)

(vi) Sulfur oxidation (e.g. Sulfurimonas)

(vii) Iron oxidation (bacteria, e.g. Acidithiobacillus, and archaea, e.g. Ferroplasma)

(viii) Iron reduction (e.g. Geobacter)

(ix) Aerobic respiration (heterotrophs, e.g. E. coli)

Species, whose complete genomes are available, were chosen to represent all major lineages of bacteria and archaea, and all the above bioenergetic modes. Information about the metabolism (bioenergetic mode) of each species was collected from the species description at the NCBI BioProject database, as well as from the Integrated Microbial Genomes database. Full details of the 198 bacteria and 74 archaea species selected are given in Table S1, while the number of species from each lineage, and each bioenergetic mode is shown in Table 1. As has been observed in previous analyses $[16,17,18]$, certain bioenergetic modes can be shared by quite distinct taxonomic groups. Indeed, as demonstrated by $16 \mathrm{~S}$ rRNA phylogenetic analysis of the organisms examined here (Figure 1), species which utilize the same bioenergetic modes do not always segregate in monophyletic groups.

Inferring the origin of each bioenergetic mode is therefore confounded by their patchy distribution among the prokaryotes. Oxygenic photosynthesis is the only bioenergetic mode which is unique to a lineage (the cyanobacteria). Oxidative phosphorylation (respiration) is shared by the greatest variety of lineages, and as such, can be considered as an ancient mode of generating energy in both the bacteria and the archaea, while methanogenesis is found in seven lineages within the euryarchaea, and as such can be considered ancient to this group However, anoxygenic photosynthesis, sulfur reduction, sulfate reduction, sulfur oxidation, iron reduction and iron oxidation are found in more than one lineage, which are not closely related. The presence of the same pathway in these distinct lineages, can come about by one of three processes: either (a) all bioenergetic modes were found in the common ancestor of these lineages, and some have been lost from some lineages, or (b) bioenergetic modes were acquired by distinct lineages by horizontal gene transfer (HGT), or (c) some electron transport chains originated multiple times independently in different lineages. The most parsimonious explanation is probably HGT, since, based on the phylogenetic tree of Figure 1, and as summarized at the bottom of Table 1, the distribution of bioenergetic pathways can be explained by up to 26 independent origins or, alternatively, 17 horizontal gene transfer events. Four HGT events can be inferred for iron oxidation, three HGT events can be inferred for anoxygenic photosynthesis, sulfate reduction, sulfur oxidation and iron reduction, and one HGT event can explain the distribution of sulfur reduction (Table 1). These inferences are based on minimal assumptions of lineage groupings (e.g. for the alpha- beta- gamma- and delta-proteobacteria) as the branching order of prokaryotic lineages is still largely unresolved $[25,26,27,28,29]$; the lineage-groupings seen in a more recent and better-resolved bacterial phylogeny [30] still do not change these numbers. Moreover, while iron reduction, and anoxygenic photosynthesis are specific to the bacteria, the other modes (sulfate reduction, sulfur reduction, sulfur oxidation, and iron 


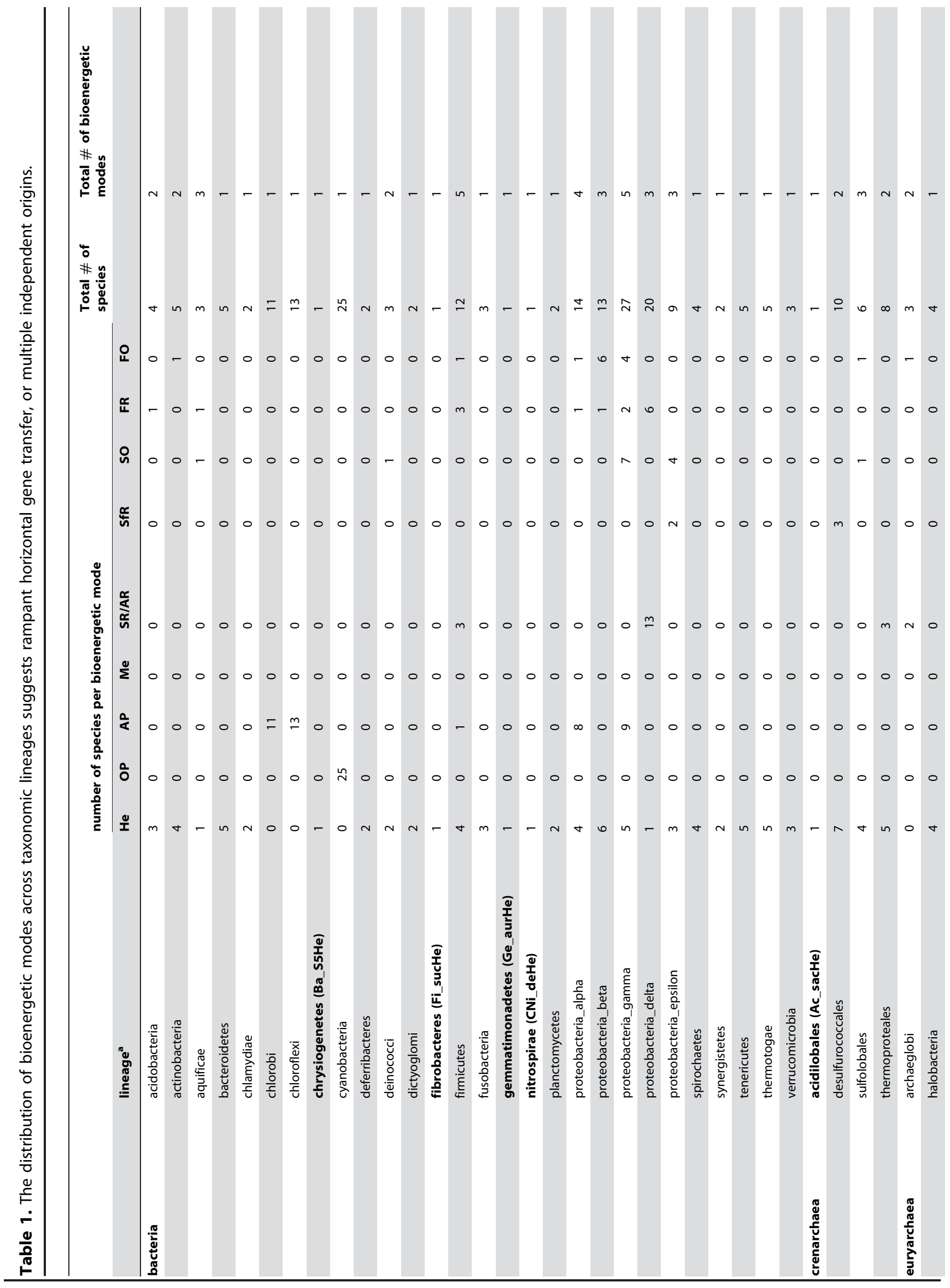




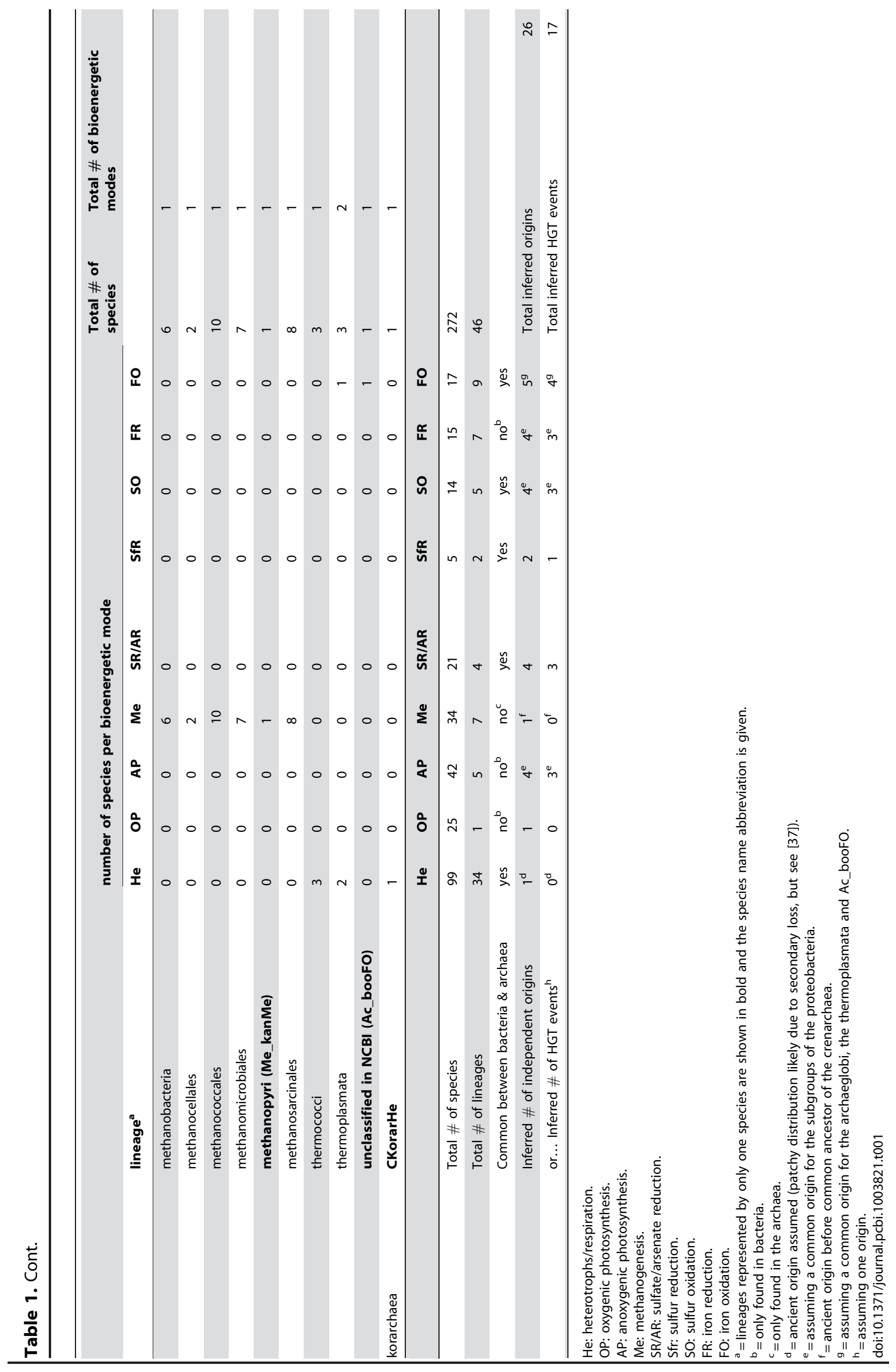




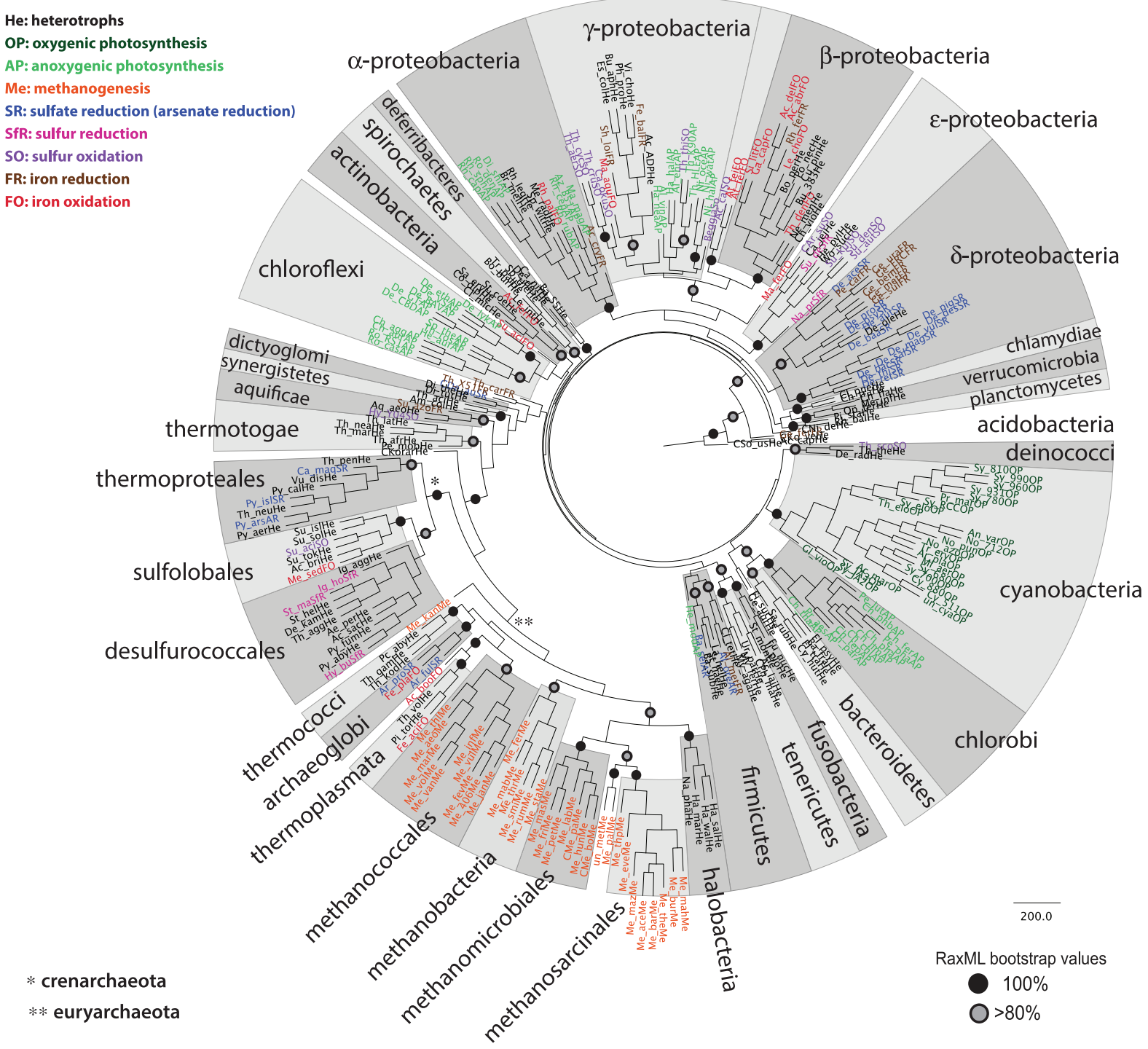

Figure 1. Phylogenetic reconstruction based on 16S rRNA sequences to map the taxonomic distribution of bioenergetic pathways. 272 prokaryotic species are shown, whose full genome sequence is available, and which represent the full diversity of bacteria and archaea, colourcoded based on their bioenergetic mode. Bootstrap values for highly supported nodes have been replaced by symbols, as indicated. The full species names, as well as details and accession numbers for all sequences used are given in Table S1. The tree shown was produced by RaxML, and its topology broadly agrees with the one produced by PhyML (the analysis based on MrBayes did not converge after 5 million generations when all sequences were included; however, when the bacteria and the archaea were examined separately, the MrBayes analysis also agreed with the RaxML and PhyML results).

doi:10.1371/journal.pcbi.1003821.g001

oxidation) are found in both bacteria and archaea. Notably, certain lineages seem more prone to bioenergetic diversity than others. For example, five bioenergetic modes are seen within the gamma-proteobacteria and the firmicutes; four bioenergetic modes are found within the alpha-proteobacteria, three bioenergetic modes are found within the beta- the delta- and the epsilonproteobacteria, the aquificae, and the sulfolobales; two bioenergetic modes are found within the deinococci, the acidobacteria, the actinobacteria, the thermoproteales, the desulfurococcales, and the thermoplasmata, while sulfate reduction and iron oxidation are both seen in the archaeoglobi. However, this may be influenced by how many complete genomes are available per lineage, and how well this represents the true diversity in each lineage [31]. This picture may thus change in the future, as more diverse organisms are sequenced.

\section{Phylogenetic analysis of the ATP synthase genes}

As the ATP synthase complex is common to all the electron transport chains of the studied bioenergetic modes, we chose to study the evolution of this complex in the different lineages. To examine whether the ATP synthase complex which is associated with the different bioenergetic modes was also subject to HGT, we performed phylogenetic analysis of all the protein subunits of the $\mathrm{F}_{0} \mathrm{~F}_{1}$ ATP synthase, as this is shared by most of the bacterial lineages. However, archaea and certain bacterial species/lineages lack $\mathrm{ATPF}_{0} \mathrm{~F}_{1}$ altogether, and have ATPV instead: Clostridium tetani and Thermoanaerobacter sp. X513 (clostridia), Chlamydia trachomatis and Chlamydophila pneumoniae (chlamydiae), Deinococcus radiodurans, Thermus scotoductus and Thermus thermophilus (deinococci), Fibrobacter succinogenes (fibrobacteres), Borrelia burgdorferi, Spirochaeta thermophila and Treponema 
He: heterotrophs

OP: oxygenic photosynthesis

AP: anoxygenic photosynthesis

Me: methanogenesis

SR: sulfate/arsenate reduction

SfR: sulfur reduction

SO: sulfur oxidation

FR: iron reduction

FO: iron oxidation

MrBayes/PhyML/RaxML

$>0.99 / 95 \% / 95 \%$

$>0.95 / 80 \% / 80 \%$

$>0.80 / 50 \% / 50 \%$

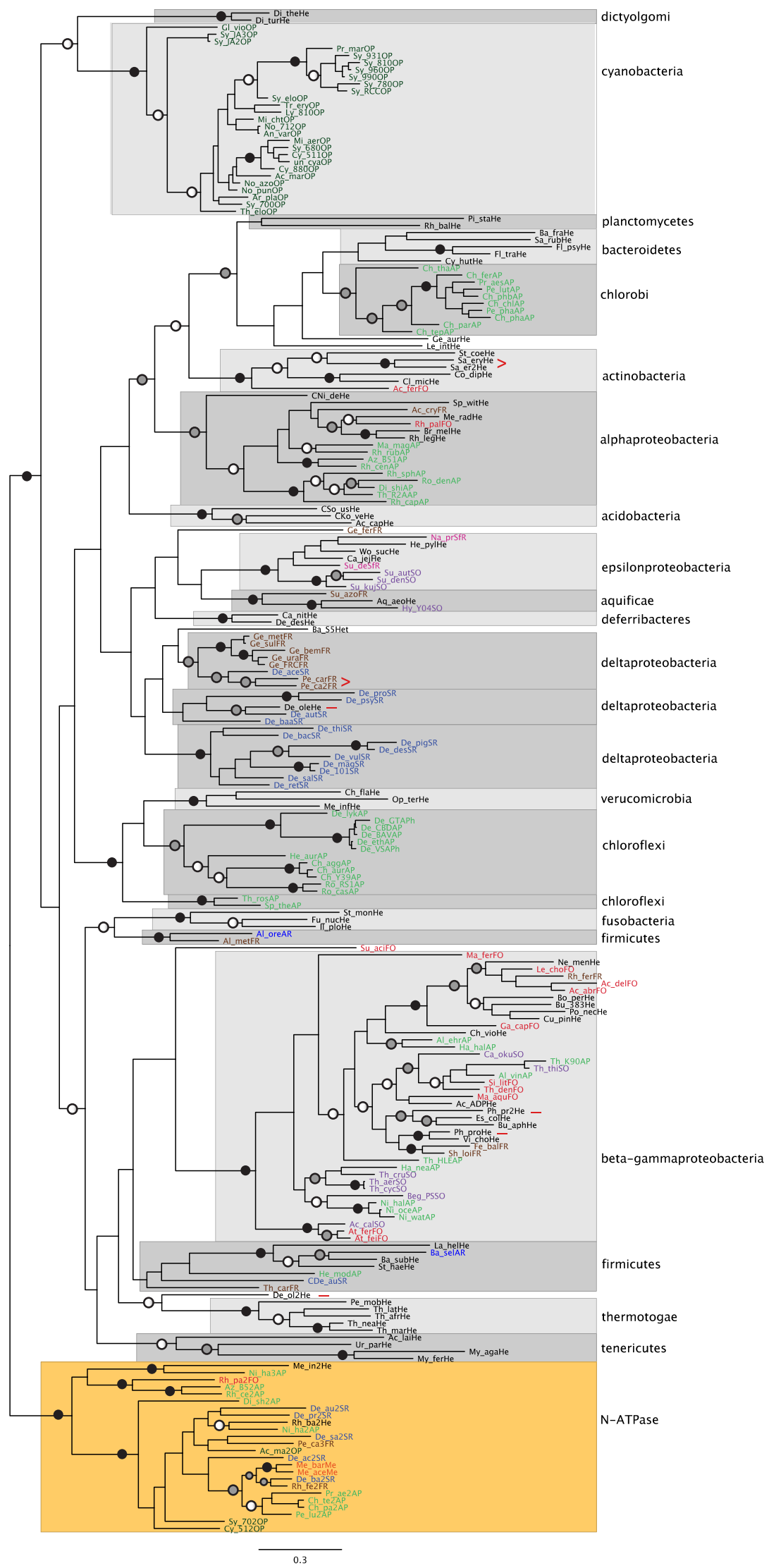


Figure 2. Phylogenetic reconstruction of ATPFOA. The tree shown is the best Bayesian topology, based on 215 sequences and 232 amino acid positions (length after trimming; median sequence length before trimming: 254$)$. Numerical values at the nodes of the tree ( $\mathrm{x} / \mathrm{y} / \mathrm{z})$ indicate statistical support by MrBayes, PhyML and RAxML (posterior probability, bootstrap and bootstrap, respectively). Values for highly supported nodes have been replaced by symbols, as indicated. Species names are colour-coded based on their bioenergetic mode as in Figure 1. Full details and accession numbers for all protein sequences used are given in Table S1. The tree is rooted at the N-ATPase clade, previously reported to be the result of horizontal gene transfer in a variety of species, all of which also contain a canonical ATPF $\mathrm{F}_{1}$ (apart from the two Methanosarcina species shown, which also have a canonical ATPV). The tree confidently separates the major bacterial taxonomic lineages, but with limited support for their branching order: strong support is provided for a subgroup containing the verrucomicrobia and chloroflexi, while another subgroup containing the alpha-proteobacteria, actinobacteria, chlorobi, bacteroidetes and planctomycetes also has reasonable support. This group also includes the spirochaete Leptospira interrogans and the gemmatimonadete Gemmatimonas aurantiaca, as well as Candidatus Nitrospira defluvii which groups with the alpha-proteobacteria. Reasonable support is also provided for the grouping of dictyoglomi and cyanobacteria, and for a subgroup containing the fusobacteria, firmicutes, tenericutes, thermotogae, and beta-gamma-proteobacteria. Two species-specific duplications (in Saccharopolyspora erythraea and Pelobacter carbinolicus) are highlighted with a red " $>$ ". Two further duplications are highlighted with a red "-" after the species name; in Photobacterium profundum the duplication either occurred before the split from other closely-related species or represents HGT from other gamma-proteobacteria; the duplication in Desulfococcus oleovorans possibly represents HGT from thermotogae (also see Figures S1 and S2). doi:10.1371/journal.pcbi.1003821.g002

pallidum (spirochaetaceae), Aminobacterium colombiense and Thermanaerovibrio acidaminovorans (synergistetes), Candidatus Phytoplasma mali (mollicutes). As most subunits of the V-type and the F-type ATPases are not homologous [24], we chose to focus solely on the $\mathrm{F}_{0} \mathrm{~F}_{1}$ ATP synthase.

Gene sequences were identified using KEGG orthology annotations, both by searching the KEGG orthology tables, and by manual searches in IMG (for the species not included in KEGG). The bacterial $\mathrm{F}_{0} \mathrm{~F}_{1} \mathrm{ATP}$ synthase complex is composed of the $\mathrm{F}_{0}$ subcomplex, which is embedded in the membrane, and the $\mathrm{F}_{1}$ subcomplex which protrudes on the side of the membrane towards which the protons exit following the proton gradient. The $\mathrm{F}_{0}$ subcomplex is composed of ATPF0A (K02108), ATPF0B (K02109), and ATPF0C (K02110), while the $\mathrm{F}_{1}$ subcomplex is composed of ATPF1A (K02111), ATPF1B (K02112), ATPF1D (K02113), ATPF1E (K02114), and ATPF1G (K02115). The genes encoding these subunits are usually arranged consecutively in a conserved genetic locus, which also includes another subunit, ATPI (K02116) and sometimes atpR. K02116 is interchangeably associated with two pfam domains, which makes orthologous gene assignments problematic: for consistency in the text below, ATPI sequences containing the pfam03899-ATP_synthI domain will be called "sI", and ATPI sequences containing the pfam09527ATPase_genel will be called "I": atpR sequences containing the pfam12966-atpR domain will be called " $\mathrm{R}$ ".

For each subunit, the corresponding protein sequences were downloaded from KEGG for all species and, after multiple alignment, phylogenetic analysis was performed using Bayesian and maximum likelihood methods. The phylogenetic analysis for ATPF0A and ATPF1A are shown in Figures 2 and 3, respectively, while the rest of the trees are in Figures S1, S2, S3, S4, S5, S6, S7. Overall, for all subunits, species segregate based on taxonomic groups with good bootstrap support, as in the $16 \mathrm{~S}$ tree, and not based on bioenergetic mode. If the current patchy distribution of bioenergetic modes (Figure 1) is due to HGT, we might expect the ATP synthase sequences from different organisms which utilize the same pathway to group together (as we used different colours for the different bioenergetic modes for species names on the tree, we would essentially expect to see organisms grouping based on colour). This is not what we observe, suggesting that there is no evidence of HGT of the ATP synthase despite the use of different bioenergetic modes between closely related species.

Nevertheless, in certain species, a duplication of the whole ATPFOF1 locus is seen (Table 2), and the majority of those duplications correspond to the so-called N-ATPase, which appears to have been acquired via horizontal gene transfer, as has been reported previously [32]. The N-ATPase genetic locus is characterized by the absence of the ATPF1D gene and the presence of the $a t p R$ gene (Figure 4) as well as a long (>100aa)
C-terminal extension in ATPF0B (Dataset S1). For the set of organisms studied here, the N-ATPase is found in certain species of planctomycetes (Rhodopirellula baltica), verrucomicrobia (Methylacidiphilum infernorum), chlorobi (Chlorobaculum parvum, Chlorobaculum tepidum (partial), Pelodictyon luteolum, Prosthecochloris aestuarii), cyanobacteria (Acaryochloris marina, Cyanothece sp. ATCG 51142, Synechococcus sp. PCG 7002), alpha-proteobacteria (Azospirillum sp. B510, Dinoroseobacter shibae, Rhodopseudomonas palustris, Rhodospirillum centenum/Rhodocista centenaria), beta-proteobacteria (Rhodoferax ferrireducens), gammaproteobacteria (Nitrosococcus halophilus - double N-ATPase, one locus split, both missing atpR), delta-proteobacteria (Desulfobacterium autotrophicum, Desulfobulbus propionicus, Desulfomicrobium baculatum, Desulfovibrio salexigens, Desulfuromonas acetoxidans, Pelobacter carbinolicus) and methanomicrobia (Methanosarcina acetivorans, Methanosarcina barkeri).

The sequences corresponding to the N-ATPase form a highly supported monophyletic group; the trees (apart from ATPF1D) were therefore rooted at this N-ATPase clade. Phylogenetic reconstruction of all subunits confidently separates the major bacterial taxonomic lineages, but the trees only give limited support for the branching order (Figures 2-3, S1, S2, S3, S4, S5, $\mathrm{S6}$ ). The differences between trees, with respect to the resolution of the branching order of different lineages, are probably due to the sequence length of the proteins analyzed; longer subunits retain more information and tend to give better-resolved phylogenetic trees, than shorter sequences [33]. The most clear-cut grouping is that of the beta- and gamma-proteobacteria, which is seen in all trees, and has significant bootstrap support in all but the ATPF1D and ATPF1E trees. Significant bootstrap support for the beta- and gamma-proteobacteria grouping is also seen in the $16 \mathrm{~S}$ phylogenetic analysis (Figure 1), which also suggests groupings of the chlorobi and the bacteroidetes, and of the fusobacteria and tenericutes. The phylogenetic link between the chlorobi and the bacteroidetes is also seen in the trees for ATPF0A (Figure 2), ATPF0C (Figure S2), ATPF1A (Figure 3) and ATFP1B (Figure S3). In the ATPFOC analysis this group also includes the planctomycetes as well as the spirochaete Leptospira interrogans and the gemmatimonadete Gemmatimonas aurantiaca (Leptospira interrogans also groups with the planctomycetes in the ATPF1A phylogeny). The ATPF0A phylogeny also has reasonable support for grouping the chlorobi, bacteroidetes and planctomycetes, together with the actinobacteria and the alpha-proteobacteria (this group also includes the spirochaete Leptospira interrogans and the gemmatimonadete Gemmatimonas aurantiaca, as well as Candidatus Nitrospira defluvii which groups with the alpha-proteobacteria; Candidatus Nitrospira defluvii also groups with the alphaproteobacteria in the ATPF0C analysis). A group containing the actinobacteria and the planctomycetes (as well as the spirochaete 
He: heterotrophs OP: oxygenic photosynthesis

AP: anoxygenic photosynthesis Me: methanogenesis

SR: sulfate/arsenate reduction

SfR: sulfur reduction

SO: sulfur oxidation

FR: iron reduction

FO: iron oxidation

MrBayes/PhyML/RaxML

$>0.99 / 95 \% / 95 \%$

$>0.95 / 80 \% / 80 \%$

$>0.80 / 50 \% / 50 \%$

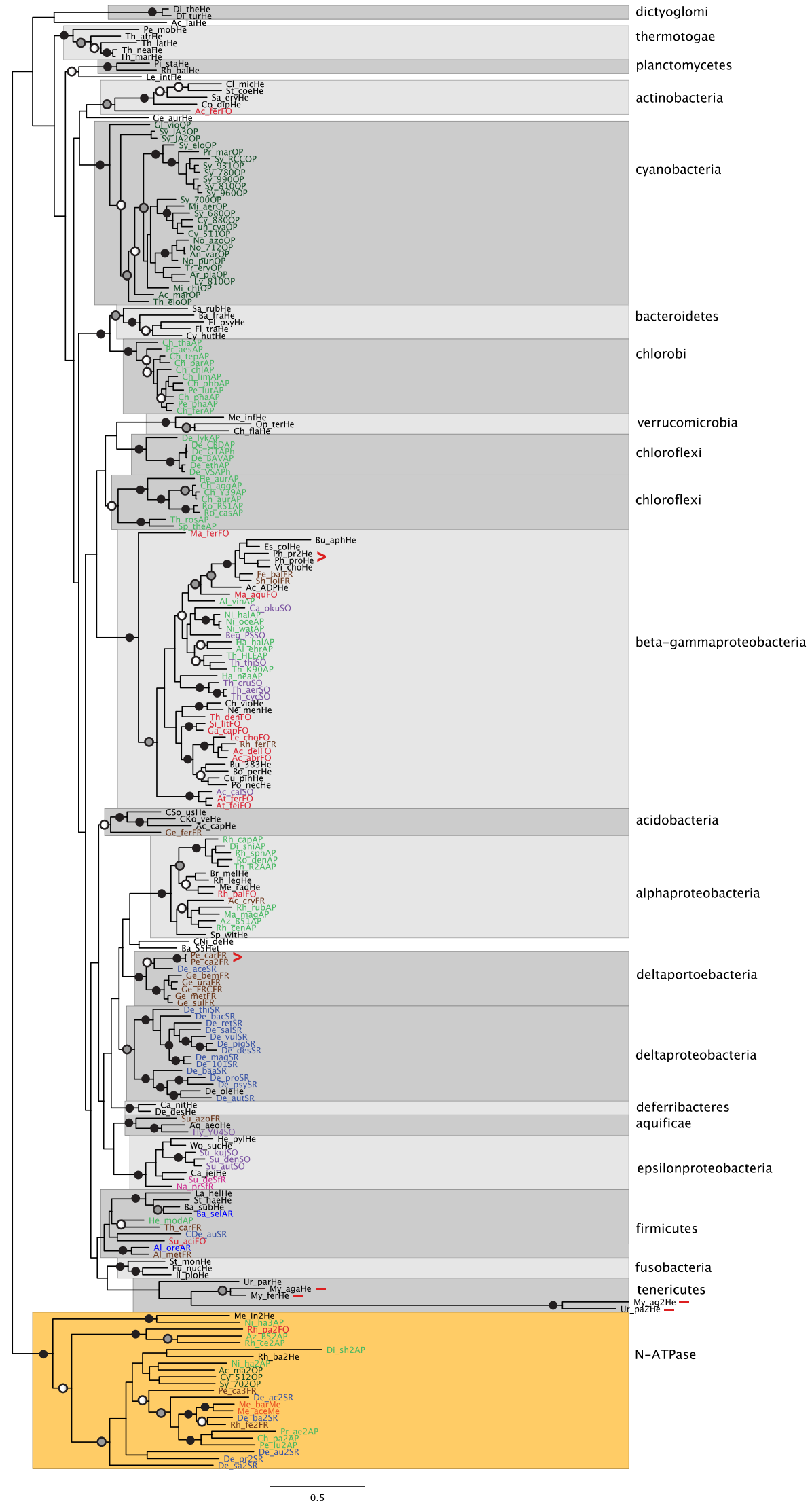


Figure 3. Phylogenetic reconstruction of ATPF1 A. The tree shown is the best Bayesian topology, based on 215 sequences and 502 amino acid positions (length after trimming; median sequence length before trimming: 508). Numerical values at the nodes of the tree ( $\mathrm{x} / \mathrm{y} / \mathrm{z})$ indicate statistical support by MrBayes, PhyML and RAxML (posterior probability, bootstrap and bootstrap, respectively). Values for highly supported nodes have been replaced by symbols, as indicated. Species names are colour-coded based on their bioenergetic mode as in Figure 1. Full details and accession numbers for all protein sequences used are given in Table S1. The tree is rooted at the N-ATPase clade, previously reported to be the result of horizontal gene transfer in a variety of species, all of which also contain a canonical ATPF $\mathrm{F}_{1}$ (apart from the two Methanosarcina species shown, which also have a canonical ATPV). The tree confidently separates the major bacterial taxonomic lineages, but with limited support for their branching order: reasonable support is only provided for one subgroup containing the chlorobi, and the bacteroidetes. The spirochaete Leptospira interrogans groups with the planctomycetes. Two species-specific duplications (in Photobacterium profundum and Pelobacter carbinolicus) are highlighted with a red " $>$ ". Two further duplications within the tenericutes are highlighted with a red "-" after the species name; this duplication likely happened before the split between Mycoplasma agalactiae and Ureaplasma parvum.

doi:10.1371/journal.pcbi.1003821.g003

Leptospira interrogans and the gemmatimonadete Gemmatimonas aurantiaca) is supported by the ATPF1G tree. Strong support is provided by the ATPF0A phylogeny for a group containing the verrucomicrobia and chloroflexi; the phylogenetic reconstruction of ATPF1G (Figure S6) also has reasonable support for a group containing the verrucomicrobia, chloroflexi, and the beta-gammaproteobacteria. Finally, reasonable support is provided in the ATPF0A tree for the grouping of dictyoglomi and cyanobacteria, and for a group containing the fusobacteria, firmicutes, tenericutes, thermotogae, and beta-gamma-proteobacteria. In the ATPFOC analysis, the dictyglomi cluster with the N-ATPase with good statistical support (Figure S2).

Although the phylogenetic analysis is based on trimmed sequences, i.e. only the unambiguous homologous regions were retained for phylogenetic analysis by manually inspecting and masking/trimming the sequences, some notable insertions/deletions were noted in the multiple alignments. For example, the chlorobi and the bacteroidetes are both missing the C-terminal half of ATPF1E, and share an internal 10-15aa insertion in ATPF1A. A different internal 10-15aa insertion in ATPF1A is shared between the beta- and gamma-proteobacteria. Actinobacteria have a $\sim 75$ aa insertion near the N-terminus of ATPF1D, and cyanobacteria have an internal 20aa insertion in ATPF1G. The N-ATPase ATPF1A in Azospirillum sp. B510 has a long $(\sim 100$ aa) $N$-terminal extension plus a $\sim 150$ aa insertion near the N-terminus, while the N-ATPase ATPF1G in Cyanothece sp. ATCG 51142 has a 50aa N-terminal extension (Dataset S1). The elucidation of the role of these signature sequences would require further study based on experimental or structural analysis.

\section{Genetic locus organization of the ATP synthase genes}

Given the ancient origin of the ATP synthase complex, the syntenic genetic location of the genes was checked in all lineages, to identify common gene order transversions, gene duplications, and possible horizontal gene transfer events (Figure 4). The NATPase, which has been suggested to be an early-diverging branch of membrane ATPases [32] has the following gene order: IB-IE-IR-0A-0C-0B-IA-IG. Bacteroides fragilis also has a similar gene locus organization, except that it lacks atpR. The subunits are arranged in consecutive order (i.e. the locus is not split) in the dictyoglomi, planctomycetes, firmicutes, thermotogae, chloroflexi, actinobacteria, tenericutes, verrucomicrobia, fusobacteria and the beta- and gamma-proteobacteria. Except for the proteobacteria and the verrucomicrobia, these lineages have been suggested to be near the base of the bacterial clade, either based on phylogenetic analysis $[25,31]$ or based on the analysis of signature sequences $[26,30]$. By inference, the most likely ancient gene order for the $\mathrm{ATPF}_{0} \mathrm{~F}_{1}$ locus is: I-sI-0A-0C-0B-ID-IA-IG-IB-IE, although some lineages lack I or sI or both (e.g. fusobacteria, chloroflexi, verrucomicrobia).

The locus has been split (indicated by semi-colons in Figure 4) at the junction between IG and IB in the chlorobi, bacteroidetes, cyanobacteria, aquificae and Beggiatoa, with further splits between IB and IE in aquificae and Beggiatoa. A further split is seen between ID and IA in the chlorobi and between IA and IG in aquificae and Beggiatoa. A split between $0 \mathrm{~B}$ and ID is seen in nitrospirae and the alpha-proteobacteria, while a split between $0 \mathrm{C}$ and $\mathrm{OB}$ is seen in aquificae, acidobacteria, deferribacteres, and delta- and epsilon-proteobacteria. A split between $0 \mathrm{~A}$ and $0 \mathrm{C}$ has occurred in the epsilon-proteobacteria. Finally a split between I and $\mathrm{OA}$ is seen in aquificae. Therefore, although there are three "blocks" of genes which are usually conserved, in terms of gene order (one containing I(-sI)-0A-0C(-0B')-0B, another containing ID-IA-IG, and another with IB-IE), in principle, gene-order transversion can and has happened all along the genetic locus.

\section{Most commonly duplicated/lost genes}

The phylogenetic analysis and the gene locus information were used to examine the most likely origin of duplicated genes, i.e. whether they arose as gene duplications within a particular species, or via horizontal gene transfer (Table 2). In the delta-proteobacterium Pelobacter carbinolicus, there are two duplications of the whole $\mathrm{ATPF}_{0} \mathrm{~F}_{1}$ locus, one corresponds to the N-ATPase, and the other is a species-specific duplication (Figures 2-3, S1, S2, S3, S4, $\mathrm{S} 5$, S6). A duplicated $\mathrm{ATPF}_{0} \mathrm{~F}_{1}$ full locus, which does not correspond to the N-ATPase was also found in the gammaproteobacterium Photobacterium profundum; this appears as a species-specific duplication in the ATPF1A, ATPF1B and ATPF1E trees (Figures 3, S3, S5), while in the rest of the trees, one copy groups with Vibrio cholerae and the other elsewhere within the gamma clade (Figure 2, S1, S2, S4, S6). This possibly hints at HGT from another closely related species, but the placement within the gamma clade is not consistent and could thus simply be due to high sequence divergence of the second copy in $P$. profundum for some of the subunits.

There are also certain in-locus gene duplications, where the duplicated genes are still found adjacent to each other on the genetic locus, as well as ectopic duplications (outside the main $\mathrm{ATPF}_{0} \mathrm{~F}_{1}$ locus) probably resulting from recombinations/transversions (summarized in Table 2). The most commonly in-locus duplicated genes are ATPF0B and ATPI, discussed in more detail in the next section. The delta-proteobacterium Desulfococcus oleovorans has a fully duplicated ectopic ATPF0 complement; in the ATPF0B phylogeny (Figure S1) both copies group within the delta-proteobacteria suggesting that this could be a species-specific duplication where one copy has diversified. However, the duplicated ATPF0A (Figure 2) and ATPF0C (Figure S2) subunits group with the thermotogae with good bootstrap support, hinting at a possible HGT event; assuming a common origin for all three subunits in the duplicated locus, this suggestion of HGT from Thermotogae requires further study (phylogenetic analysis of only the deltaproteobacteria and thermotogae sequences did not resolve this issue as it gives the same results as above for the duplicated subunits, data not shown). The actinobacterium 

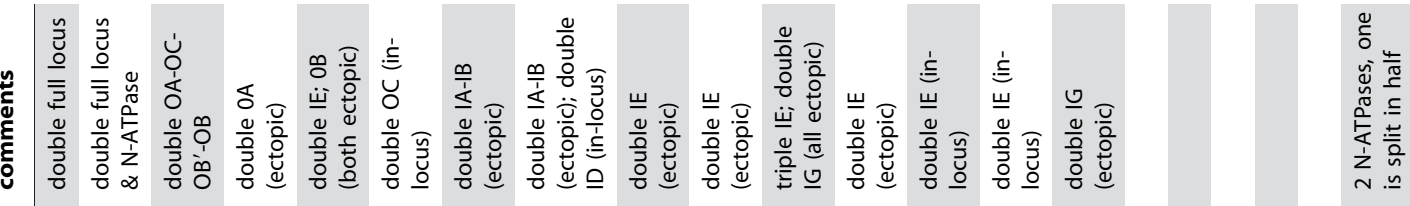

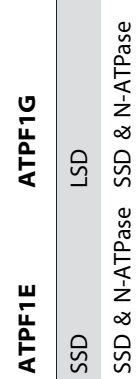
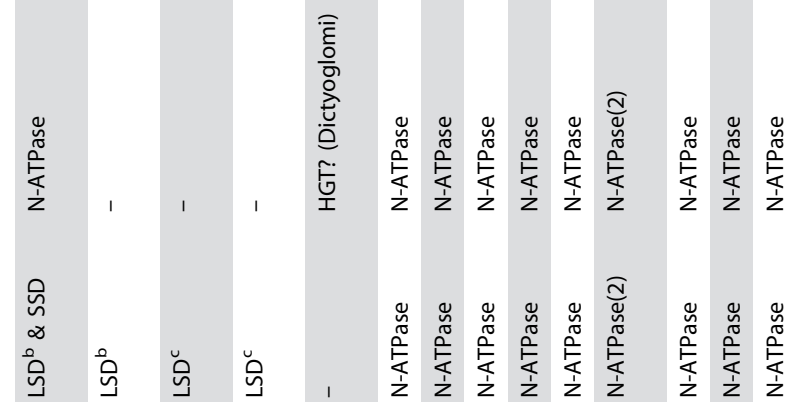

号

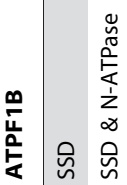

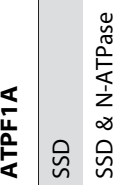

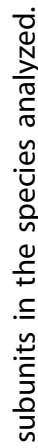

宽

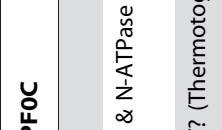

产

ติ

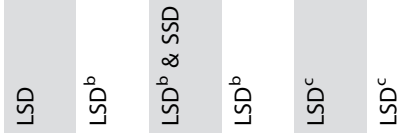

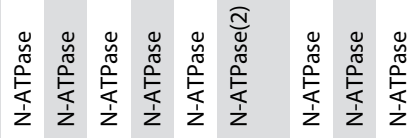

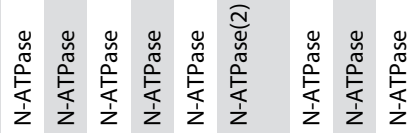

点 毫

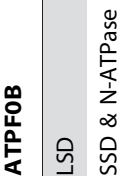

乌ิ

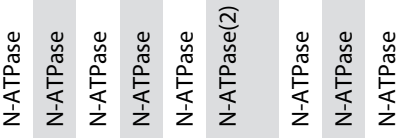

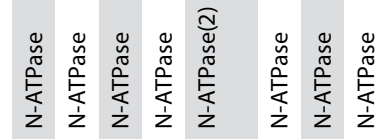

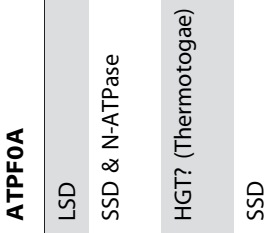

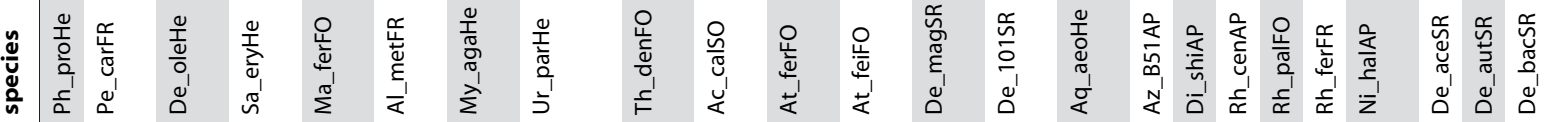

范

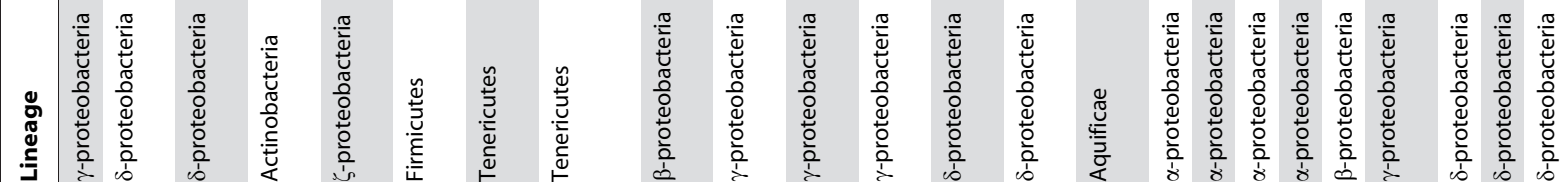




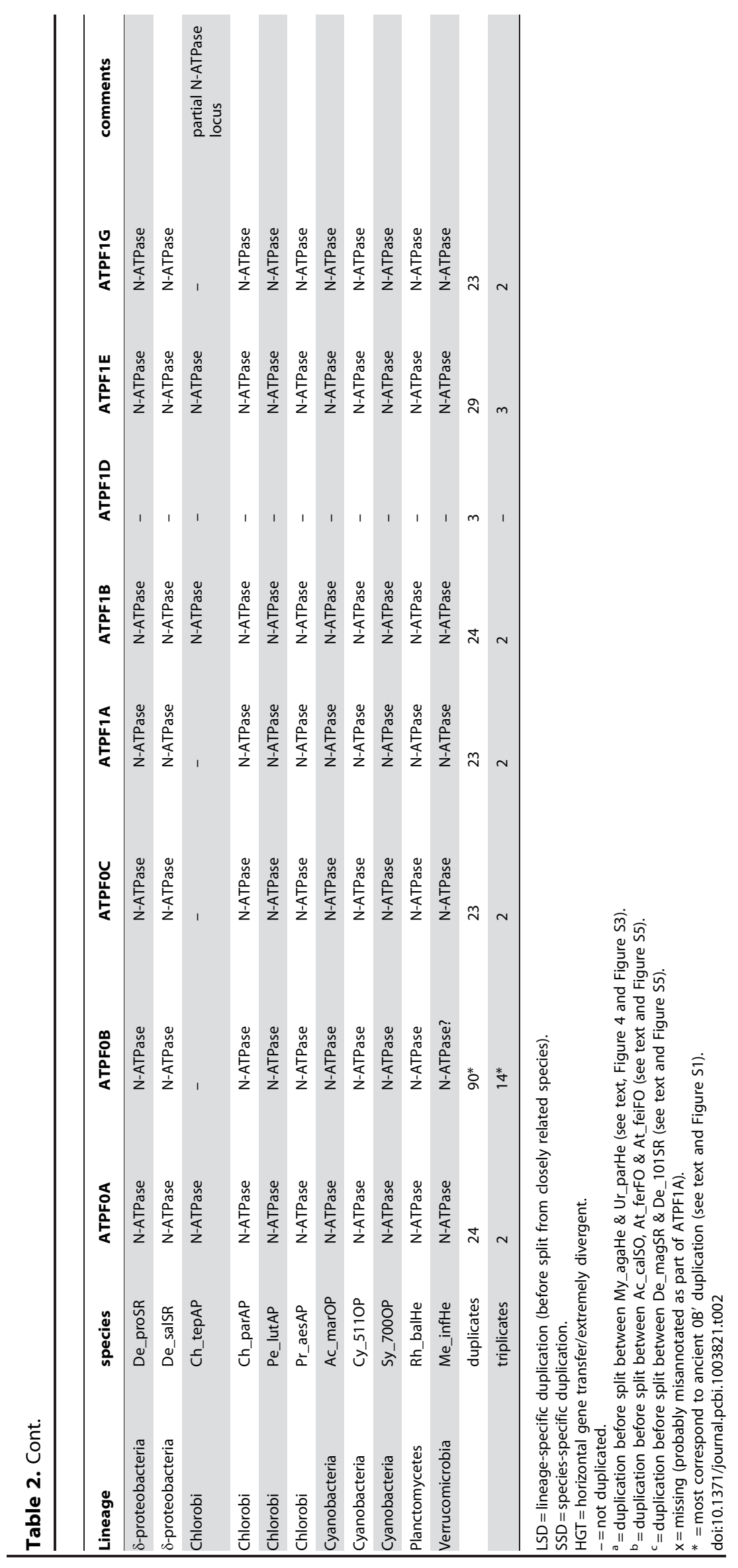


N-ATPase (missing ID):

Dictyoglomi (missing ID):

Planctomycetes*:

Firmicutes*/Thermotogae*:

Chloroflexi (He_aurAP and Chloroflexales missing I):

Actinobacteria*/Tenericutes*:

Verrucomicrobia (missing l/sl):

Fusobacteria/Beta-gammaproteobacteria: (Po_necHe, Bu_aphHe missing sl) Ha_neaAP, Th_aerSO, Th_cycSO, Th_cruSO:

Be_PSSO:

Chlorobi:

Bacteroidetes*:

Ba_fraHe:

Nitrospirae (CNi_deHe):

Gemmatimonadetes (Ge_aurHe):

Cyanobacteria (Mi_chtOP missing IB-IE):

Sy_JA2OP, Sy_JA3OP:

Th_eloOP, GI_vioOP:

Aquificae (Su_azoFR):

(Hy_Y04SO):

Aq_aeoHe (missing I):

Alpha-proteobacteria (Ac_cryFR missing I):

Epsilon-proteobacteria:

Delta-proteobacteria/Acidobacteria*/

Deferribacteres/ Chrysiogenetes (Ba_S5He):

Pe_carFR (extra gene locus):
IB-IE-I-R-OA-0C-OB-IA-IG

I-sI(?)-0A-0C-0B-IA-IG-IB-IE

$|-s|(?)-0 A-0 C-0 B-|D-| A-|G-| B-\mid E$

|-s|-0A-0C-0B-ID-IA-IG-IB-IE

I-OA-0C-0B-ID-IA-IG-IB-IE

I(?)-OA-OC-OB-ID-IA-IG-IB-IE

OA-0C-0B-ID-IA-IG-IB-IE

sI-0A-0C-0B-ID-IA-IG-IB-IE

I-sI-0A-0C-0B-ID-IA-IG-IB-IE

SI-X-OA-OC-OB-ID-IA; IG; IB(2); IE

I-sI(?)-0A-0C-0B-ID; IA-IG; IB-IE

I-sI(?)-0A-0C-0B-ID-IA-IG; IB-IE

IB-IE- I(?)-OA-0C-0B-ID-IA-IG

I-OA-0C-0B; ID-IA-IG-IB-IE

I-sI(?)-0A-0C-0B-ID; IA-IG-IB-IE

sI-0A-0C-0B'-0B-ID-IA-IG; IB-IE

sl-0A-0C-0B'-0B-ID-IA-IG; IB; IE

sI-OA-0C-0B'-OB-ID-IA; IG; IB; IE

OA-X-0C; 0B'-0B-ID-IA-IG-IB; IE

I; OA-X-OC; OB'-0B-ID-IA; IG; IB; IE

OA-X-OC; OB'-OB-ID; IA; IG-IB; IE

I-OA-OC-0B'-0B; ID-IA-IG-IB-IE

I; 0A; 0C; 0B'-OB-ID-IA-IG-IB-IE

I-sI-OA-0C; 0B'-0B-ID-IA-IG-IB-IE

OB'-OB-ID-IA-IG-IB-IE-OA-OC

Figure 4. $\mathbf{A T P F}_{\mathbf{0}} \mathbf{F}_{\mathbf{1}}$ gene locus organization per lineage. The $A T P F_{0} F_{1}$ gene locus organization was checked for all species in the IMG database [47], and is summarized per lineage. The gene order shown follows the order in which the genes are transcribed in each genome (upstream to downstream). Semicolons indicate that the separated gene groups are on non-adjacent genetic locations (and can be very far upstream or downstream; e.g. separated by only 4 intervening ORFs in Geobacter sp. FRC-32, and by up to 5026 intervening ORFs, or 6 Mb, in Nostoc sp. PCC 7120; see Table S1). When the locus is split, the genes are shown in the order they are usually found in when the locus is intact. ATPFOB (K02109) is often duplicated, so one copy is called OB, and the other OB", based on the gene order. ATPI (K02116) is also often duplicated, and is designated "I" "sl" and " $\mathrm{R}$ " based on the presence of distinct pfam domains, as discussed in the text. Question marks indicate that the ATPI subunit is sometimes not clearly 
assigned to the orthology group. " $\mathrm{X}$ " denotes hypothetical intervening ORFs. Notable variations within some lineages are shown. *Especially for lineages represented by relatively few species, please see TableS1 for variations between the species examined within each lineage. doi:10.1371/journal.pcbi.1003821.g004

Saccharopolyspora erythraea has a duplicated ectopic ATPF0A, which looks like a species-specific duplication (Figure 2). The zetaproteobacterium Mariprofundus ferrooxydans has a duplicated ectopic ATPF0B; it is unclear if this is the result of HGT, as the sequence groups with planctomycetes, but not with high bootstrap support (Figure S1). The firmicute Alkaliphilus metalliredigens has a species-specific in-locus duplication of ATPF0C (Figure S2) which is characterized by a long $(\sim 100$ aa $)$ N-terminal extension (Dataset S1). Ectopic duplications of ATPF1A and ATPF1B are seen in Mycoplasma agalactiae and Ureaplasma parvum (tenericutes) as has been reported recently [34]; this duplication likely happened before the split between the two species (Figure 3, S3); one of the ATPF1A copies in $U$. parvum has a long ( $\sim 250$ aa) $\mathrm{C}$ terminal extension (Dataset S1). Ureaplasma parvum also has duplicated ATPF1D in-locus; the evolutionary history of this duplication cannot be clearly inferred from the phylogenetic analysis, although it appears to be species-specific in the PhyML and RaxML trees, but is not statistically supported by high bootstrap values. ATPF1E is duplicated ectopically in Mariprofundus ferrooxydans (zeta-proteobacteria), Thiobacillus denitrificans (beta-proteobacteria), and the gamma-proteobacteria Acidithiobacillus caldus, Acidithiobacillus ferrivorans, and Acidithiobacillus ferrooxidans (two extra copies), as well as in-locus in the delta-proteobacteria Desulfovibrio magneticus and Desulfovibrio sp. FW1012B. The duplication in M. ferrooxydans is speciesspecific, while the other duplications are lineage-specific, i.e. the duplication either occurred before the split from other closelyrelated species or represents HGT from other closely-related species (Figure S5): the duplication in $T$. denitrificans may represent HGT from other gamma-proteobacteria; a duplication occurred before the split between the gamma-proteobacteria Acidithiobacillus ferrooxidans, Acidithiobacillus ferrivorans and Acidithiobacillus caldus, with a further species-specific duplication in Acidithiobacillus ferrooxidans; another duplication occurred before the split between Desulfovibrio magneticus and Desulfovibrio sp. FW1012B in the delta-proteobacteria. ATPF $1 \mathrm{G}$ is ectopically duplicated in Aquifex aeolicus (aquificae; one copy has a 80aa C-terminal extension) and Acidithiobacillus ferrooxidans (gamma-proteobacteria; one copy is missing the N-terminal half); the duplication in Aquifex aeolicus represents a very divergent sequence which groups with the dictyoglomi in the MrBayes and PhyML trees (Figure S6); the duplication in $A$. ferrooxidans might be a pseudogene as it is much smaller in size in the tree it clusters with the N-ATPase genes.

ATPF0B is duplicated in-locus in acidobacteria, aquificae, cyanobacteria, deferribacteres, and alpha- delta- and epsilonproteobacteria. This raises the question of whether ATPF0B has been duplicated independently in separate lineages, or whether the duplication has been passed on, either by direct descent, or by horizontal gene transfer. In the phylogenetic analysis (Figure S1) the ATPF0B' group in the alpha-proteobacteria appears as a sister group to the alpha-proteobacterial ATPF0B, but with only moderate statistical support (red asterisk: 0.7 posterior probability in MrBayes, 50\%, and 46\% bootstrap support in PhyML and RaxML, respectively). The other ATPF0B's group together (blue asterisk), with good statistical support by MrBayes (posterior probability: 1) but with low support in PhyML and RaxML (24\% and $26 \%$ bootstrap support, respectively). The grouping of the alpha-proteobacterial ATPFOB and ATPF0B' may indicate that this duplication happened more recently than the ATPF0B duplications in the other lineages. However, given the low bootstrap support it remains unclear from the tree whether the ATPF0B $/ 0 \mathrm{~B}^{\prime}$ duplication happened independently in the different lineages where it is observed, or whether it happened only once in the common ancestor of all the lineages where it is observed (and presumably lost in other lineages, e.g. the beta-gamma-proteobacteria); however, the latter scenario is more plausible based on parsimony considerations.

Notable absences are the ATPF1D in N-ATPase, as well as in dictyoglomi (Dictyoglomus thermophilum and Dictyoglomus turgidum), ATPFOC in Wolinella succinogenes (epsilon-proteobacteria), ATPF1B and ATPF1E in the cyanobacterium Microcoleus chthonoplastes, and ATPI missing from many species (e.g. chloroflexales, verrucomicrobia). At least some of these absences may of course be due to incomplete annotation or extreme sequence divergence.

\section{Evolution of atpl, sl, and R}

ATPI has been the least studied subunit of the $\mathrm{F}_{0} \mathrm{~F}_{1}$ ATP synthase complex. As mentioned above, ATPI (K02116) is interchangeably associated with two pfam domains (pfam03899ATP_synthI and pfam09527-ATPase_gene1), which makes orthologous gene assignments problematic. The bacterial uncI gene encoding a small transmembrane protein which includes the pfam03899 domain, has been demonstrated to have a chaperone role in assisting the assembly of the c-ring of the $\mathrm{F}_{0}$ subcomplex $[35,36]$. By analogy, it has been suggested that the atpR gene of the N-ATPase (characterized by the presence of the pfam12966 domain) plays a similar role, in the absence of uncI [32]. Given this suggestion, and the grouping of the $a t p Q$ genes (which include the pfam09527 domain) into the same KEGG cluster as uncI, along with the fact that all three encode proteins of similar size and, based on their position in the genetic cluster, could be the result of gene duplications, we decided to analyze their evolutionary relationship in more detail.

The phylogenetic reconstruction of ATPI (K02116) protein sequences, including "sI" proteins containing the pfam03899ATP_synthI domain, "I" proteins containing the pfam09527ATPase_genel, and " $\mathrm{R}$ " proteins containing the pfam12966-atpR domain (found in the N-ATPase locus) is shown in Figure S7. Overall the three types of proteins look similar in the alignment, although atpR stands out, as do the cyanobacterial sI sequences; the delta-proteobacterium Desulfovibrio piger has a prominent 50 aa C-terminal extension (Dataset S1). Only the PhyML tree is shown, even though the bootstrap support for most branches is not significant. Phylogenetic analysis with the same set of sequences using MrBayes failed to converge on a tree, and the RaxML tree had very bad resolution. The low resolution and low bootstrap support are probably due to the short sequence length and high divergence of these sequences. Nevertheless, the tree does separate a cluster of the "I" proteins (which contain the pfam09527ATPase_genel domain) to the left of the dotted grey line, and another cluster containing the "sI" proteins (pfam03899-ATP_ synthI domain) and "R" proteins (pfam12966-atpR domain) to the right of the grey dotted line. Based on the gene locus organization and the protein sizes, the genes encoding the "sI" and " $\mathrm{R}$ " proteins look like duplications of the "I" gene, and the tree indeed supports this hypothesis. However, due to the low resolution of the phylogenetic analysis, the issue of the origin and functional 
homology of atpI, sI, and R would ultimately need to be resolved with structural and functional analysis.

\section{Discussion}

Phylogenetic analysis of 16S rRNA for 272 species chosen to represent all the major prokaryotic lineages and bioenergetic modes indicated that, overall, there is no monophyly of bioenergetic modes (one notable exception is oxygenic photosynthesis which is confined to the cyanobacteria). This analysis also highlighted lineages which include species with vastly different modes of generating energy (e.g. proteobacteria, firmicutes). The scattered distribution of certain bioenergetic modes, such as anoxygenic photosynthesis or iron oxidation, indicates rampant HGT of at least some bioenergetic modes, in agreement with previous analyses $[16,17,18]$. All these bioenergetic pathways also include the ATP synthase complex, but phylogenetic analysis of all the $\mathrm{ATPF}_{0} \mathrm{~F}_{1}$ synthase subunits, common to almost all bacterial lineages, largely agree with the $16 \mathrm{~s}$ rRNA tree. This indicates that, if different bioenergetic pathways dispersed into different lineages by horizontal gene transfer, this did not involve the ATP synthase complex. Presumably, each species used its pre-existing ATP synthase complex and adapted it to utilize the proton gradient generated by vastly different ETCs. Recent data has shown that large-scale HGT from bacteria transformed the bioenergetic capabilities of the Haloarchaea [37] and yet Haloarchaea retain ATPV, whereas their laterally acquired bioenergetics modes utilize ATPF in the bacteria. This is in agreement with our results, and again indicates flexibility in combining a species' pre-existing ATP synthase with a newly acquired electron transport chain. Given the widespread effect of HGT on prokaryotic evolution [38,39,40], it may be that the cost of incorporating a laterally transferred ATP synthase to replace a pre-existing enzyme is too high to overcome [41]. To our knowledge the question of whether specific modifications are needed for the ATP synthase to function with different bioenergetic modes has not been addressed previously, so this current, updated large-scope study allows us to resolve this issue, and suggests that no apparent such modifications exist, at least at the sequence level. A more thorough structural analysis would be needed to examine if certain structural modifications unite the ATP synthases of organisms using each bioenergetic pathway.

HGT has happened however, for a variant form of the ATP synthase, previously named N-ATPase, as it includes residues in the c subunit for translocating $\mathrm{Na}^{+}$[32]. This is found always in addition to the $\mathrm{F}_{0} \mathrm{~F}_{1}$ ATP synthase, in certain species from different bacterial lineages, as well as in two Methanosarcina species of the archaea. The N-ATPase subunits always cluster independently of their $\mathrm{F}_{0} \mathrm{~F}_{1}$ counterparts, and although they often group closest to the dictyoglomi, only the ATPF0C phylogeny has significant bootstrap support for a grouping of the dictyoglomi and $\mathrm{N}$-ATPase; therefore, their exact origin cannot be inferred from the tree, and possibly predates the separation between ATPV and ATPF [32]. The N-ATPase locus is characterized by the absence of the ATPF1D subunit, and the presence of the atpR gene (also see below). Interestingly, the two dictyoglomi species studied here (the only two for which complete genome information is available) also lack the ATPF1D subunit, which in combination with the close affinity of the dictyoglomi and the N-ATPase in most of the trees, might suggest that the dictyoglomi are the closest relative to the common ancestor of the N-ATPase. In the gammaproteobacterium Nitrosococcus halophilus, two copies of the $\mathrm{N}$ ATPase are found (one locus is split in half, both are missing atpR), whereas Chlorobaculum tepidum of the chlorobi only has half the locus; the lack of certain subunits may indicate a non-functional degenerate N-ATPase. It is assumed that the N-ATPase confers a selective advantage in high-salt environments [32].

Given the ancient origin of the $\mathrm{F}_{0} \mathrm{~F}_{1}$ ATPase, the phylogenetic trees can perhaps give clues as to the evolutionary relationships between different bacterial lineages. The branching order of bacterial lineages remains an issue unresolved through phylogenetic analysis [25,27,28,29], although other methods have also been proposed based on signature sequences of certain crucial proteins [26], and a more recent analysis based on feature frequency profiles in whole proteome data has produced a wellresolved tree [30]. Some of the $\mathrm{F}_{0} \mathrm{~F}_{1}$ ATP synthase subunits are relatively long proteins, and relatively slow evolving due to their interactions with the other subunits, so they may retain some of the evolutionary signal that cannot be retrieved from $16 \mathrm{~S}$ rRNA sequences. There is consistent support for a grouping of the betaand gamma-proteobacteria, another of the chlorobi and the bacteroidetes, and some support for this group also including the planctomycetes, the actinobacteria, the alpha-proteobacteria and the spirochaete Leptospira interrogans and the gemmatimonadete Gemmatimonas aurantiaca; Candidatus Nitrospira defluvii groups with the alpha-proteobacteria. Some trees also indicate a subgroup containing the verrucomicrobia and the chloroflexi, and possibly also the beta-gamma-proteobacteria. Finally, reasonable support is provided in the ATPF0A tree for the grouping of dictyoglomi and cyanobacteria, and for a subgroup containing the fusobacteria, tenericutes, firmicutes, thermotogae, and beta-gamma-proteobacteria. The groupings of (i) the beta-gamma proteobacteria, (ii) the chlorobi and bacteroidetes, and (iii) the fusobacteria, tenericutes, firmicutes, and thermotogae, are in agreement with the more recent phylogeny [30].

The order of the genes encoding the $\mathrm{F}_{0} \mathrm{~F}_{1}$ ATP synthase subunits is relatively well conserved overall in most of the species analyzed, although the locus has been split on multiple occasions, and the genes for ATPF1B and ATPF1E are found either upstream (in the N-ATPase and in Bacteroides fragilis) or, most commonly, downstream of all the others. Duplications of each of the $\mathrm{F}_{0} \mathrm{~F}_{1} \mathrm{ATP}$ synthase subunits are observed in several species, either within the genetic locus or in distant parts of the genome. The history of these duplications can be traced by looking at the phylogenetic analysis. The most ancient in-locus duplication is likely that of atpI, with the diversification of the downstream copy into "sI" and "R", but with multiple losses in various lineages of either one or both copies. Another ancient in-locus duplication is that of ATPFOB, which probably occurred in the common ancestor of the acidobacteria, aquificae, cyanobacteria, deferribacteres, delta- epsilon- and alpha-proteobacteria, (and presumably lost in other lineages, e.g. the beta-gamma-proteobacteria). Most of the other duplications have occurred in isolated species, and appear to be species-specific, with no unassailable evidence of HGT.

These duplications raise the question of how certain species deal with gene dosage effects, e.g. to co-ordinate the ATP synthase complex structure. As there is no clear evidence of HGT, apart from the N-ATPase clade, most duplications seem to be the result of stochastic events that have not been bred out; presumably this means that at least some of these duplications, e.g. the ATPF0B/ $0 \mathrm{~B}^{\prime}$ duplication may confer a selective advantage, although this would need to be confirmed experimentally. A recent study of the ATPV complex showed that such paralogous expansions can lead to increased complexity (and possibly also specificity) of a multisubunit molecular machine [42]. Moreover, ATPF0B functions as a dimer, even in species where only one copy exists in the genome, and the two parts of the dimer interact with different parts of the 
$\mathrm{F}_{1}$ and the $\mathrm{F}_{0}$ subcomplex $[43,44]$. Thus a gene duplication which allows each gene copy to fine-tune specific interactions may indeed be advantageous. Notably, cyanobacterial ATPF0B/0B' have been successfully inserted into a null $E$. coli strain (which lacks its native single ATPFOB) and form heterodimers which assemble with the rest of the native ATP synthase $E$. coli subunits to form a functional enzyme [45]. This again points to a flexibility of the ATP synthase in different species, to accommodate changes and duplications.

The loss of ATPF1D from N-ATPase and dictyoglomi, as well as ATPI from many species also raises the question of the essentiality of these subunits for the function of the $\mathrm{F}_{0} \mathrm{~F}_{1}$ ATP synthase. The absence of certain subunits in isolated species (ATPF0G from Wolinella succinogenes (epsilon-proteobacteria), ATPF1B and ATPF1E from the cyanobacterium Microcoleus chthonoplastes) may be due to incomplete annotation or extreme sequence divergence, although if they represent true losses, again this raises questions as to the functionality of the ATP synthase in these species.

Overall, this analysis highlights the patchy distribution of bioenergetic modes across prokaryotic lineages, which is most likely the result of HGT. However, there is no evidence of HGT for the ATP synthase to accompany the spread of bioenergetic pathways in different lineages. This means that the ATP synthase cannot be used to reconstruct the origin of the diversity of bioenergetic modes in prokaryotes. It also indicates that there are no apparent specific modifications of the $\mathrm{F}_{0} \mathrm{~F}_{1}$ ATP synthase in order for it to work with different bioenergetic ETCs. The $\mathrm{F}_{0} \mathrm{~F}_{1}$ ATP synthase genetic locus is overall well conserved, although as demonstrated by multiple splits and duplications, in principle, the system is robust and flexible, as it can deal with a split between any subunits and/or a duplication of any subunit. The elucidation of the way in which certain species deal with these duplications, splits and losses, and the advantage any of these may confer, now requires further study.

\section{Materials and Methods}

\section{Organism selection}

Bacteria and archaea species, whose genomes have been completely sequenced and are available at NCBI, were chosen by parsing the NCBI Genome Project database (http://www.ncbi. nlm.nih.gov/bioproject) with keywords relating to the relevant metabolisms (e.g. "anoxygenic phototroph"), and the relevant phyla (e.g. "chlorobi”). For autotrophs and chemolithotrophs, all relevant species were examined, but for heterotrophs, only a sample of species was examined so as to cover the full diversity of bacteria and archaea [31] (http://tolweb.org/tree/) and the full bioenergetic diversity per lineage. For lineages with many sequenced genomes, the tree of [31] was used to pick species so as to cover as much phylogenetic diversity as possible with a limited number of species. The set of species selected, represent 131 clusters, with a genome similarity score (GSS) threshold of 0.5 ; of those, 24 are in "clusters" which only have one member, and 63 are the sole representatives from their cluster [46]. Information on the metabolic mode of all species was also cross-checked in the IMG database [47]. Each species name was assigned an 8character abbreviation for better data handling during the phylogenetic analysis, by keeping the first two letters of the first name and the first three letters of the second name, as well as a $2-$ 3 letter ending, denoting the bioenergetic mode. Details of all the 272 organisms analyzed, and of the species names abbreviations are given in Table $\mathrm{S} 1$.

\section{Sequence retrieval and phylogenetic analysis}

16S rRNA sequences were downloaded pre-aligned from the RDP database [48]. When more than one sequence was available for each species/strain examined, one of the good-quality $>$ $1200 \mathrm{bp}$ sequences was selected at random, unless the type sequence was available, in which case that was selected. Importantly, we used data from the same strain for the $16 \mathrm{~S}$ analysis and the ATP analysis (see below). As bacterial and archaeal sequences are provided as separate pre-aligned files, the program opal was used to align the two sets [49]. Common gaps were removed after manual examination of the whole set of sequences in McClade. The nucleotide substitution model that best fits the data $(\mathrm{GTR}+\mathrm{I}+\mathrm{G})$ was selected using the program ModelGenerator [50] (http://bioinf.nuim.ie/modelgenerator/).

All other analyses were done at the amino acid level. For the ATP synthase subunits, sequence accession numbers were retrieved using the ortholog tables from the KEGG database: KEGG ortholog tables are based on RefSeq annotations, sequence similarity and best-hit searches, as well as tools for operon-like consistency and completeness of pathway modules and complexes; furthermore they are regularly updated (http://www.kegg.jp/ $\mathrm{kegg} / \mathrm{ko.html}$ ). In cases where data was missing from the KEGG database, this was supplemented by data from IMG [47], manual analysis to find the best reciprocal BLAST hits, as well as synteny considerations, since the gene order of the ATP synthase locus is well-conserved overall. The accession numbers of all sequences analyzed, and the corresponding species names abbreviations, are given in Table S1. Sequences were downloaded from KEGG in fasta format using a custom perl script. Alignments were created using MUSCLE [51]. Only unambiguous homologous regions were retained for phylogenetic analysis by manually inspecting and masking/trimming the sequences in McClade (the masked alignment are given in Dataset S1). ProtTest [52] was used to estimate the appropriate model of sequence evolution.

Phylogenetic analysis was performed by three separate methods. To obtain the Bayesian tree topology and posterior probability values, the program MrBayes version 3.1.2 was used [53]. Analyses were run for 1-5 million generations, removing all trees before a plateau established by graphical estimation. All calculations were checked for convergence and had a splits frequency of $<0.1$. Maximum-likelihood (ML) analysis was performed using PhyML [54] and RAxML [55] with 100 bootstrap replicates. Nodes with better than 0.95 posterior probability and $80 \%$ bootstrap support were considered robust, and nodes with better than 0.80 posterior probability and $50 \%$ bootstrap support are shown. Tree files were processed in Figtree vl.4 and Adobe Illustrator to highlight homologous groups, and colour-code species names based on bioenergetic mode.

\section{Genetic locus analysis}

As the genes encoding the different subunits of the ATP synthase are normally clustered in an operon, the genetic locus of the sequences analyzed was examined in the IMG database [47]. Details of the locus organization in each species are given in Table $\mathrm{S} 1$ and the data is summarized per lineage in Figure 4.

\section{Supporting Information}

Figure S1 Phylogenetic reconstruction of ATPF0B. The tree shown is the best Bayesian topology, based on 298 sequences and 161 amino acid positions (length after trimming; median sequence length before trimming: 170). Numerical values at the nodes of the tree $(\mathrm{x} / \mathrm{y} / \mathrm{z})$ indicate statistical support by MrBayes, PhyML and RAxML (posterior probability, bootstrap and 
bootstrap, respectively). Values for highly supported nodes have been replaced by symbols, as indicated. Species names are colourcoded based on their bioenergetic mode. Full details and accession numbers for all protein sequences used are given in Table $\mathrm{S} 1$. The tree is rooted at the N-ATPase clade, previously reported to be the result of horizontal gene transfer in a variety of species, all of which also contain a canonical $\mathrm{ATPF}_{0} \mathrm{~F}_{1}$ (apart from the two Methanosarcina species shown which also have a canonical ATPV). The tree confidently separates the major bacterial taxonomic lineages, but with no clear support for their branching order; notably however, the dictyglomi cluster with the N-ATPase with good statistical support. There are multiple duplications, most of which represent the in-locus duplication of ATPF0B/0B' seen in acidobacteria, aquificae, cyanobacteria, deferribacteres, deltaepsilon- and alpha-proteobacteria. The ATPF0B' group in the alpha-proteobacteria appears as a sister group to the alphaproteobacterial ATPF0B, although with only moderate statistical support (red asterisk: 0.7 posterior probability in MrBayes, $50 \%$, and 46\% bootstrap support in PhyML and RaxML, respectively). The other ATPF0B's group together (blue asterisk), with good statistical support by MrBayes (posterior probability: 1) but with low support by PhyML and RaxML (24\% and 26\% bootstrap support, respectively). It is thus unclear from the tree whether the ATPF0B/0B' duplication happened independently in the different lineages where it is observed, or whether it happened only once in the common ancestor of all the lineages where it is observed (and presumably lost in other lineages, e.g. the beta-gamma-proteobacteria); however, the latter scenario is more plausible based on parsimony considerations. Two species-specific duplications in Pelobacter carbinolicus are highlighted with a red " $>$ ". Four more duplications are highlighted with a red "-" after the species name: in Photobacterium profundum the duplication either occurred before the split from other closely-related species or represents HGT from other gamma-proteobacteria; in Desulfococcus oleovorans the duplication (which is a duplication of the full ATPF0 locus) seems to be species-specific, contrary to what is seen for ATPF0A in Figure 2 and ATPF0C in Figure S2; it is unclear if the duplication in the zeta-proteobacterium Mariprofundus ferrooxydans is the result of HGT, as the sequence groups with planctomycetes, but not with high bootstrap support. The duplication in Methylacidiphilum infernorum (highlighted with a yellow "_" after the species name) represents the ATPF0B within the N-ATPase locus, but it did not group with the other N-ATPase ATPF0Bs, probably due to its long branch length.

(EPS)

Figure S2 Phylogenetic reconstruction of ATPF0C. The tree shown is the best Bayesian topology, based on 214 sequences and 77 amino acid positions (length after trimming; median sequence length before trimming: 81). Numerical values at the nodes of the tree $(\mathrm{x} / \mathrm{y} / \mathrm{z})$ indicate statistical support by MrBayes, PhyML and RAxML (posterior probability, bootstrap and bootstrap, respectively). Values for highly supported nodes have been replaced by symbols, as indicated. Species names are colourcoded based on their bioenergetic mode. Full details and accession numbers for all protein sequences used are given in Table S1. The tree is rooted at the N-ATPase clade, previously reported to be the result of horizontal gene transfer in a variety of species, all of which also contain a canonical $\mathrm{ATPF}_{0} \mathrm{~F}_{1}$ (apart from the two Methanosarcina species shown which also have a canonical ATPV). The tree confidently separates the major bacterial taxonomic lineages, but with limited support for their branching order: reasonable support is only provided for one subgroup containing the chlorobi, bacteroidetes and planctomycetes (as well as the spirochaete Leptospira interrogans and the gemmatimonadete Gemmatimonas aurantiaca). Candidatus $\mathrm{Ni}$ trospira defluvii groups with the alpha-proteobacteria. Two species-specific duplications (in Pelobacter carbinolicus and Alkaliphilus metalliredigens) are highlighted with a red " $>$ ". Two further duplications are highlighted with a red "-" after the species name; in Photobacterium profundum the duplication either occurred before the split from other closely-related species or represents HGT from other gamma-proteobacteria; the duplication in Desulfococcus oleovorans possibly represents HGT from thermotogae (also see Figure 2).

(EPS)

Figure S3 Phylogenetic reconstruction of ATPF1B. The tree shown is the best Bayesian topology, based on 215 sequences and 458 amino acid positions (length after trimming; median sequence length before trimming: 470). Numerical values at the nodes of the tree $(\mathrm{x} / \mathrm{y} / \mathrm{z})$ indicate statistical support by MrBayes, PhyML and RAxML (posterior probability, bootstrap and bootstrap, respectively). Values for highly supported nodes have been replaced by symbols, as indicated. Species names are colourcoded based on their bioenergetic mode. Full details and accession numbers for all protein sequences used are given in Table S1. The tree is rooted at the N-ATPase clade, previously reported to be the result of horizontal gene transfer in a variety of species, all of which also contain a canonical $\mathrm{ATPF}_{0} \mathrm{~F}_{1}$ (apart from the two Methanosarcina species shown which also have a canonical ATPV). The tree confidently separates the major bacterial taxonomic lineages, but with limited support for their branching order: reasonable support is only provided for one subgroup containing the chlorobi and the bacteroidetes. Two species-specific duplications (in Photobacterium profundum and Pelobacter carbinolicus) are highlighted with a red " $>$ ". Two further duplications within the tenericutes are highlighted with a red "--" after the species name; this duplication likely happened before the split between Mycoplasma agalactiae and Ureaplasma parvum. (EPS)

Figure S4 Phylogenetic reconstruction of ATPF1D. The tree shown is the best Bayesian topology, based on 189 sequences and 180 amino acid positions (length after trimming; median sequence length before trimming: 181). Numerical values at the nodes of the tree $(\mathrm{x} / \mathrm{y} / \mathrm{z})$ indicate statistical support by MrBayes, PhyML and RAxML (posterior probability, bootstrap and bootstrap, respectively). Values for highly supported nodes have been replaced by symbols, as indicated. Species names are colourcoded based on their bioenergetic mode. Full details and accession numbers for all protein sequences used are given in Table S1. The tree is rooted at Thermotogae, which is generally accepted as being one of the ancestral lineages of the bacteria (N-ATPase has no ATPF1D). The tree confidently separates the major bacterial taxonomic lineages, but with no clear support for their branching order. One species-specific duplication is highlighted with a red " $>$ " in Pelobacter carbinolicus. Two further duplications are highlighted with a red "_" after the species name: in Photobacterium profundum the (lineage-specific) duplication either occurred before the split from other closely-related species or represents HGT from other gamma-proteobacteria; the evolutionary history of the duplication in Ureaplasma parvum cannot be clearly inferred from the phylogenetic analysis; it appears to be speciesspecific in the PhyML and RaxML trees, but is not statistically supported by high bootstrap values.

(EPS)

Figure S5 Phylogenetic reconstruction of ATPF1E. The tree shown is the best Bayesian topology, based on 221 sequences 
and 138 amino acid positions (length after trimming; median sequence length before trimming: 137). Numerical values at the nodes of the tree $(\mathrm{x} / \mathrm{y} / \mathrm{z})$ indicate statistical support by MrBayes, PhyML and RAxML (posterior probability, bootstrap and bootstrap, respectively). Values for highly supported nodes have been replaced by symbols, as indicated. Species names are colourcoded based on their bioenergetic mode. Full details and accession numbers for all protein sequences used are given in Table $\mathrm{S} 1$. The tree is rooted at the N-ATPase clade, previously reported to be the result of horizontal gene transfer in a variety of species, all of which also contain a canonical $\mathrm{ATPF}_{0} \mathrm{~F}_{1}$ (apart from the two Methanosarcina species shown which also have a canonical ATPV). The tree confidently separates the major bacterial taxonomic lineages, but with no clear support for their branching order. Three species-specific duplications are highlighted with a red " $>$ " in Pelobacter carbinolicus, Photobacterium profundum, and Mariprofundus ferrooxydans. Lineage-specific duplications (the duplication either occurred before the split from other closelyrelated species or represents HGT from other closely-related species) are highlighted with a red "-" after the species name: one duplication seems to have occurred before the split between Desulfovibrio magneticus and Desulfovibrio sp. FW1012B in the delta-proteobacteria; another duplication is seen in the betaproteobacterium Thiobacillus denitrificans (which may represent HGT from other gamma-proteobacteria); finally a duplication occurred before the split between the gamma-proteobacteria Acidithiobacillus ferrooxidans, Acidithiobacillus ferrivorans and Acidithiobacillus caldus, with a further species-specific duplication in Acidithiobacillus ferrooxidans.

(EPS)

Figure S6 Phylogenetic reconstruction of ATPF1G. The tree shown is the best Bayesian topology, based on 215 sequences and 291 amino acid positions (length after trimming; median sequence length before trimming: 291). Numerical values at the nodes of the tree $(\mathrm{x} / \mathrm{y} / \mathrm{z})$ indicate statistical support by MrBayes, PhyML and RAxML (posterior probability, bootstrap and bootstrap, respectively). Values for highly supported nodes have been replaced by symbols, as indicated. Species names are colour-coded based on their bioenergetic mode. Full details and accession numbers for all protein sequences used are given in Table S1. The tree is rooted at the N-ATPase clade, previously reported to be the result of horizontal gene transfer in a variety of species, all of which also contain a canonical $\mathrm{ATPF}_{0} \mathrm{~F}_{1}$ (apart from the two Methanosarcina species shown which also have a canonical ATPV). The tree confidently separates the major bacterial taxonomic lineages, but with limited support for their branching order: reasonable support is only provided for one subgroup containing the chloroflexi, beta-gamma-proteobacteria and the verrucomicrobia, and another subgroup containing the actinobacteria and the planctomycetes (as well as the spirochaete Leptospira interrogans and the gemmatimonadete Gemmatimonas aurantiaca). One species-specific duplication in Pelobacter carbinolicus is highlighted with a red " $>$ ". Two further duplications are highlighted with a red "_" after the species name; in Photobacterium profundum the duplication either occurred before the split from other closely-related species or represents HGT from other gamma-proteobacteria; the duplication in Aquifex aeolicus represents a very divergent sequence

\section{References}

1. Schafer G, Engelhard M, Muller V (1999) Bioenergetics of the Archaea. Microbiol Mol Biol Rev 63: 570-620. which groups with the dictyoglomi in the MrBayes and PhyML trees.

(EPS)

Figure S7 Phylogenetic reconstruction of ATPI (K02116). The analysis is based on 275 sequences and 98 amino acid positions (length after trimming; median sequence length before trimming: 114), including "sI" proteins containing the pfam03899-ATP_synthI domain, "I" proteins containing the pfam09527-ATPase_genel, and " $\mathrm{R}$ " proteins containing the pfam12966-atpR domain (found in the N-ATPase locus). Species names are colour-coded based on their bioenergetic mode. Full details and accession numbers for all protein sequences used are given in Table S1. The tree shown is based on PhyML analysis, as phylogenetic analysis with the same set of sequences using MrBayes failed to converge on a tree, and the RaxML tree had very bad resolution. In contrast to the trees for the other subunits, the bootstrap support for most branches is not significant; the low resolution and low bootstrap support are probably due to the short sequence length and high divergence of the ATPI sequences. Nevertheless, the tree does separate a cluster of the "I" proteins (which contain the pfam09527-ATPase_genel domain) to the left of the dotted grey line, and another cluster containing the "sI" proteins (pfam03899-ATP_synthI domain) and "R" proteins (pfam12966-atpR domain) to the right of the grey dotted line. Based on the gene locus organization and the protein sizes, the genes encoding the "sI" and "R" proteins look like duplications of the "I" gene, and the PhyML tree indeed supports this hypothesis. (EPS)

Table S1 Details of the species used in this study, including species name abbreviations, and accession numbers of the sequences used for the phylogenetic analyses.

(XLS)

Dataset S1 Masked alignments are provided for all the sequence datasets used to construct the trees presented in the manuscript. Each file is for one dataset and is named based on the corresponding $\mathrm{ATPF}_{0} \mathrm{~F}_{1}$ subunit. The files are in nexus format, which can be viewed either as simple text, or with a variety of programs that support nexus files (e.g. McClade). The last row of each alignment, entitled "mask" has an "I" for each position which was included in the final trimmed alignment, used in the phylogenetic analysis. Gaps in the alignment which are not marked with "I" were manually removed in McClade. (ZIP)

\section{Acknowledgments}

We are grateful to Joel Dacks and Derek Bendall for insightful discussions and for critically reviewing the manuscript. We also thank Ioanna Karamichali for providing the custom perl script used for collecting the sequences in fasta format from the KEGG database. We are indebted to the CamGrid computational resource on which most of the phylogenetic analyses were performed, and to the various genome centres and projects for open access to data for analysis.

\section{Author Contributions}

Conceived and designed the experiments: VLK SK. Performed the experiments: VLK. Analyzed the data: VLK. Wrote the paper: VLK. Critically revised the manuscript for important intellectual content: SK.

2. Deppenmeier U (2002) Redox-driven proton translocation in methanogenic Archaea. Cell Mol Life Sci 59: 1513-1533. 
3. Blankenship RE (2002) Molecular Mechanisms of Phtosynthesis. Oxford: Blackwell Science.

4. Shima S, Warkentin E, Thauer RK, Ermler U (2002) Structure and function of enzymes involved in the methanogenic pathway utilizing carbon dioxide and molecular hydrogen. J Biosci Bioeng 93: 519-530.

5. Wirsen CO, Sievert SM, Cavanaugh CM, Molyneaux SJ, Ahmad A, et al. (2002) Characterization of an autotrophic sulfide-oxidizing marine Arcobacter sp. that produces filamentous sulfur. Appl Environ Microbiol 68: 316-325.

6. Matias PM, Pereira IA, Soares CM, Carrondo MA (2005) Sulphate respiration from hydrogen in Desulfovibrio bacteria: a structural biology overview. Prog Biophys Mol Biol 89: 292-329.

7. Bryant DA, Frigaard NU (2006) Prokaryotic photosynthesis and phototrophy illuminated. Trends Microbiol 14: 488-496.

8. Weber KA, Achenbach LA, Coates JD (2006) Microorganisms pumping iron: anaerobic microbial iron oxidation and reduction. Nat Rev Microbiol 4: 752764.

9. Tamazawa S, Takasaki K, Tamaki H, Kamagata Y, Hanada S (2012) Metagenomic and biochemical characterizations of sulfur oxidation metabolism in uncultured large sausage-shaped bacterium in hot spring microbial mats. PLoS One 7: e49793.

10. Magalon A, Arias-Cartin R, Walburger A (2012) Supramolecular organization in prokaryotic respiratory systems. Adv Microb Physiol 61: 217-266.

11. Schoepp-Cothenet B, van Lis R, Atteia A, Baymann F, Capowiez L, et al. (2013) On the universal core of bioenergetics. Biochim Biophys Acta 1827: 79-93.

12. Todar K The Diversity of Procaryotic Metabolism. Online Textbook of Bacteriology. http://www.textbookofbacteriology.net/ Accessed 22 January 2014 .

13. Banfield JF, Marshall CR (2000) Perspectives: earth science and evolution. Genomics and the geosciences. Science 287: 605-606.

14. Battistuzzi FU, Feijao A, Hedges SB (2004) A genomic timescale of prokaryote evolution: insights into the origin of methanogenesis, phototrophy, and the colonization of land. BMC Evol Biol 4: 44.

15. Knoll AH, Bauld J (1989) The evolution of ecological tolerance in prokaryotes. Trans R Soc Edinb Earth Sci 80: 209-223.

16. Castresana J (2001) Comparative genomics and bioenergetics. Biochim Biophys Acta 1506: 147-162.

17. Reysenbach AL, Shock E (2002) Merging genomes with geochemistry in hydrothermal ecosystems. Science 296: 1077-1082.

18. Boucher Y, Douady CJ, Papke RT, Walsh DA, Boudreau ME, et al. (2003) Lateral gene transfer and the origins of prokaryotic groups. Annu Rev Genet 37: 283-328.

19. Baymann F, Lebrun E, Brugna M, Schoepp-Cothenet B, Giudici-Orticoni MT, et al. (2003) The redox protein construction kit: pre-last universal common ancestor evolution of energy-conserving enzymes. Philos Trans R Soc Lond B Biol Sci 358: 267-274

20. Olson JM, Blankenship RE (2004) Thinking about the evolution of photosynthesis. Photosynthesis Research 80: 373-386.

21. Sadekar S, Raymond J, Blankenship RE (2006) Conservation of distantly related membrane proteins: photosynthetic reaction centers share a common structural core. Mol Biol Evol 23: 2001-2007.

22. Cross RL, Muller V (2004) The evolution of A-, F-, and V-type ATP synthases and ATPases: reversals in function and changes in the H+/ATP coupling ratio. FEBS Lett 576: 1-4.

23. Lewalter K, Muller V (2006) Bioenergetics of archaea: ancient energy conserving mechanisms developed in the early history of life. Biochim Biophys Acta 1757: 437-445.

24. Mulkidjanian AY, Makarova KS, Galperin MY, Koonin EV (2007) Inventing the dynamo machine: the evolution of the F-type and V-type ATPases. Nat Rev Microbiol 5: 892-899.

25. Hugenholtz P, Goebel BM, Pace NR (1998) Impact of culture-independent studies on the emerging phylogenetic view of bacterial diversity. J Bacteriol 180: 4765-4774.

26. Gupta RS, Griffiths E (2002) Critical issues in bacterial phylogeny. Theor Popul Biol 61: 423-434.

27. Gao L, Qi J, Sun J, Hao B (2007) Prokaryote phylogeny meets taxonomy: an exhaustive comparison of composition vector trees with systematic bacteriology. Sci China C Life Sci 50: 587-599.

28. Bapteste E, Susko E, Leigh J, Ruiz-Trillo I, Bucknam J, et al. (2008) Alternative methods for concatenation of core genes indicate a lack of resolution in deep nodes of the prokaryotic phylogeny. Mol Biol Evol 25: 83-91.
29. Lang JM, Darling AE, Eisen JA (2013) Phylogeny of bacterial and archaeal genomes using conserved genes: supertrees and supermatrices. PLoS One 8: e62510.

30. Jun SR, Sims GE, Wu GA, Kim SH (2010) Whole-proteome phylogeny of prokaryotes by feature frequency profiles: An alignment-free method with optimal feature resolution. Proc Natl Acad Sci U S A 107: 133-138.

31. Wu D, Hugenholtz P, Mavromatis K, Pukall R, Dalin E, et al. (2009) A phylogeny-driven genomic encyclopaedia of Bacteria and Archaea. Nature 462: 1056-1060.

32. Dibrova DV Galperin MY, Mulkidjanian AY (2010) Characterization of the NATPase, a distinct, laterally transferred Nat-translocating form of the bacterial F-type membrane ATPase. Bioinformatics 26: 1473-1476.

33. Wortley AH, Rudall PJ, Harris DJ, Scotland RW (2005) How much data are needed to resolve a difficult phylogeny?: case study in Lamiales. Syst Biol 54: 697-709.

34. Beven L, Charenton C, Dautant A, Bouyssou G, Labroussaa F, et al. (2012) Specific evolution of F1-like ATPases in mycoplasmas. PLoS One 7: e38793.

35. Ozaki Y, Suzuki T, Kuruma Y, Ueda T, Yoshida M (2008) UncI protein can mediate ring-assembly of c-subunits of FoF1-ATP synthase in vitro. Biochem Biophys Res Commun 367: 663-666.

36. Suzuki T, Ozaki Y, Sone N, Feniouk BA, Yoshida M (2007) The product of uncI gene in F1Fo-ATP synthase operon plays a chaperone-like role to assist c-ring assembly. Proc Natl Acad Sci U S A 104: 20776-20781.

37. Nelson-Sathi S, Dagan T, Landan G, Janssen A, Steel M, et al. (2012 Acquisition of 1,000 eubacterial genes physiologically transformed a methanogen at the origin of Haloarchaea. Proc Natl Acad Sci U S A 109: 2053720542.

38. Dagan T, Artzy-Randrup Y, Martin W (2008) Modular networks and cumulative impact of lateral transfer in prokaryote genome evolution. Proc Natl Acad Sci U S A 105: 10039-10044.

39. Kunin V, Ouzounis CA (2003) The balance of driving forces during genome evolution in prokaryotes. Genome Res 13: 1589-1594.

40. Treangen TJ, Rocha EP (2011) Horizontal transfer, not duplication, drives the expansion of protein families in prokaryotes. PLoS Genet 7: e1001284.

41. Baltrus DA (2013) Exploring the costs of horizontal gene transfer. Trends Ecol Evol 28: 489-495.

42. Finnigan GC, Hanson-Smith V, Stevens TH, Thornton JW (2012) Evolution of increased complexity in a molecular machine. Nature 481: 360-364.

43. Brandt K, Maiwald S, Herkenhoff-Hesselmann B, Gnirss K, Greie JC, et al. (2013) Individual interactions of the $\mathrm{b}$ subunits within the stator of the Escherichia coli ATP synthase. J Biol Chem 288: 24465-24479.

44. Gajadeera CS, Weber J (2013) Escherichia coli F1Fo-ATP synthase with a b/ delta fusion protein allows analysis of the function of the individual b subunits. J Biol Chem 288: 26441-26447.

45. Claggett SB, Grabar TB, Dunn SD, Cain BD (2007) Functional incorporation of chimeric b subunits into F1Fo ATP synthase. J Bacteriol 189: 5463-5471.

46. Moreno-Hagelsieb G, Wang Z, Walsh S, ElSherbiny A (2013) Phylogenomic clustering for selecting non-redundant genomes for comparative genomics. Bioinformatics 29: 947-949.

47. Markowitz VM, Chen IM, Palaniappan K, Chu K, Szeto E, et al. (2012) IMG: the Integrated Microbial Genomes database and comparative analysis system. Nucleic Acids Res 40: D115-122.

48. Cole JR, Wang Q, Cardenas E, Fish J, Chai B, et al. (2009) The Ribosomal Database Project: improved alignments and new tools for rRNA analysis. Nucleic Acids Res 37: D141-145.

49. Wheeler TJ, Kececioglu JD (2007) Multiple alignment by aligning alignments. Bioinformatics 23: i559-568.

50. Keane TM, Creevey CJ, Pentony MM, Naughton TJ, McLnerney JO (2006) Assessment of methods for amino acid matrix selection and their use on empirical data shows that ad hoc assumptions for choice of matrix are not justified. BMC Evol Biol 6: 29.

51. Edgar RC (2004) MUSCLE: multiple sequence alignment with high accuracy and high throughput. Nucleic Acids Res 32: 1792-1797.

52. Abascal F, Zardoya R, Posada D (2005) ProtTest: selection of best-fit models of protein evolution. Bioinformatics 21: 2104-2105.

53. Ronquist F, Huelsenbeck JP (2003) MrBayes 3: Bayesian phylogenetic inference under mixed models. Bioinformatics 19: 1572-1574.

54. Guindon S, Gascuel O (2003) A simple, fast, and accurate algorithm to estimate large phylogenies by maximum likelihood. Syst Biol 52: 696-704.

55. Stamatakis A (2006) RAxML-VI-HPC: maximum likelihood-based phylogenetic analyses with thousands of taxa and mixed models. Bioinformatics 22: 26882690 . 